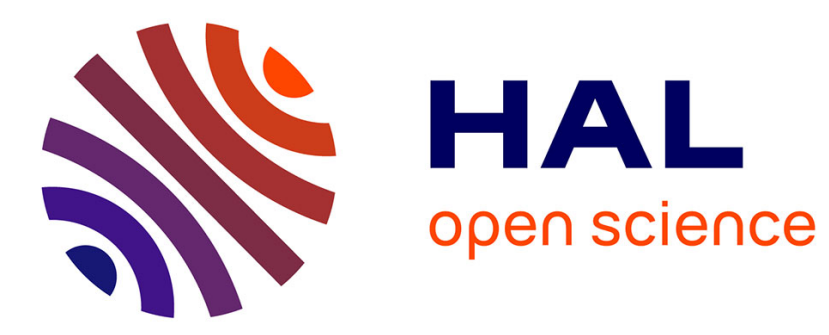

\title{
The vibration signature of chordal cracks in a rotor system including uncertainties
}

Jean-Jacques Sinou, Béatrice Faverjon

\section{To cite this version:}

Jean-Jacques Sinou, Béatrice Faverjon. The vibration signature of chordal cracks in a rotor system including uncertainties. Journal of Sound and Vibration, 2012, 331, pp.138-154. 10.1016/j.jsv.2011.08.001 . hal-00630417

\section{HAL Id: hal-00630417 \\ https://hal.science/hal-00630417}

Submitted on 25 Sep 2012

HAL is a multi-disciplinary open access archive for the deposit and dissemination of scientific research documents, whether they are published or not. The documents may come from teaching and research institutions in France or abroad, or from public or private research centers.
L'archive ouverte pluridisciplinaire HAL, est destinée au dépôt et à la diffusion de documents scientifiques de niveau recherche, publiés ou non, émanant des établissements d'enseignement et de recherche français ou étrangers, des laboratoires publics ou privés. 


\title{
The vibration signature of chordal cracks in a rotor system including uncertainties
}

\author{
Jean-Jacques Sinou ${ }^{a}$ and Béatrice Faverjon ${ }^{b}$ \\ ${ }^{a}$ Laboratoire de Tribologie et Dynamique des Systèmes UMR-CNRS 5513 \\ Ecole Centrale de Lyon, 36 avenue Guy de Collongue \\ 69134 Ecully Cedex, France \\ ${ }^{b}$ Laboratoire de Mécanique des Contacts et des Structures UMR-CNRS 5259 \\ INSA-Lyon, 18-20, rue des Sciences \\ 69621 Villeurbanne Cedex France
}

\begin{abstract}
The aim of this paper is to investigate the effects of the presence of a transverse crack in a rotating shaft under uncertain physical parameters in order to obtain some indications that might be useful in detecting the presence of a crack in rotating system. The random dynamic response of the cracked rotor is evaluated by expanding the changing stiffness of the crack (i.e. the breathing mechanism) as a random truncated Fourier series. To avoid the use of the Monte Carlo simulations (MCS), an alternative procedure that is based on a combination of the Harmonic Balance Method and the Stochastic Finite Element Method (SFEM) using the Polynomial Chaos Expansion (PCE) is proposed. So the response of the Fourier components of the cracked rotor is expanded in the polynomial chaoses. The random dynamic response obtained by applying this procedure is compared with that evaluated through numerical integration based on the Harmonic Balance Method and the Monte Carlo simulations.
\end{abstract}

\section{Introduction}

The effect of crack in rotors is important to ensure the integrity of structural components for a wide range of engineering applications. In recent years a lot of effort has been devoted to the detection of transverse cracks in shafts [1-5]. In most of the studies for crack detection in mechanical or rotor systems, researchers used changes in natural frequencies [6] and evolution of the non-linear behaviour of the system at the super-harmonics components as the diagnostic tools. For example, Gash $[1,7]$ demonstrated that a slight decrease in the natural frequencies and the appearance of new resonances when the rotational speeds of the rotor reach $\frac{1}{2}, \frac{1}{3}$ and $\frac{1}{4}$ of the resonant frequencies of the rotor are key indicators for the detection of transverse cracks in a rotating shaft. He also suggested that the non-linear behavior of the damage rotor and the opening and closing of the crack during its rotation are due mainly to the shaft's self-weight. Then, Sinou [8] indicated that the vibration amplitudes in the $\frac{1}{n}$ sub-critical resonances (with $n \geq 2$ ) depend not only on the rotor damping, unbalance, position and depth of the crack, but also on the combinations of the unbalance and the crack parameters. The sensitivity of the magnitudes of $\frac{1}{2}$ and $\frac{1}{3}$ sub-critical resonances with respect to the unbalance angle 
and the unbalance-crack interactions are different in the vertical and horizontal directions. However, the author suggested that the magnitudes of the sub-critical resonance peaks do not greatly change if the crack effect is predominant. Adewusi and Al-Bedoor $[9,10]$ investigated an experimental study on the non-linear dynamic response of an overhung rotor with a propagating transverse crack. They observed that the appearance of second harmonic component is the first signature of the presence of damage. It was demonstrated that the third harmonic components are excited just before fracture, and so can not be used as an efficient and robust factor for the detection of damage in early stages. Some researchers $[9,11-14]$ proposed to introduce time-scale signal processing tools based on wavelet transform for the detection of damage in rotating machinery due to the limitation of the conventional Fourier analysis that is suitable for steady state vibration signals, but provides a poor representation of transient signals due to a strong increase of the shaft's rotating speed.

Due to the fact that crack breathes when the system rotates, the cracked rotor has a time-dependent coefficient. The amount of open part of the crack varies with the rotation of the shaft, thereby changing the stiffness of the cracked rotor. Gash $[1,7]$ also suggested that the opening and closing of the crack during its rotation are due mainly to the shaft's self-weight. Researchers highlight the possibility of crack detection through the observation of the non-linear dynamic behaviour of rotor systems due to the this breathing mechanism [15, 15-20]. They concluded that the primary response characteristic resulting from the changes in the non-linear dynamical behaviour of the rotor system through half resonance speeds appears to be the characteristic signature for detecting the presence of a crack rotor. They also indicated that emerging of the 3X super-harmonic frequency components and the distortion of the orbit, and formation of a double loop and inside loop in the orbit [8], may provide useful information on the presence of a crack and may be used on an on-line crack monitoring rotor system. For example, Darpe [21] studied both numerically and experimentally the evolution of orbital patterns during the passage through sub-critical resonances. The authors explained that the inner loop is present at one-half of the first resonant speed due to dominant second harmonic component. When the rotor is passing through the $\frac{1}{2}$ sub-critical speed, the inner loop changes its orientation by almost 180 degrees. Moreover, Darpe et al. [22] used impulse axial excitation to a rotating cracked shaft to detect damage. They indicated that the presence of damage induces not only the coupling mechanism in lateral and longitudinal vibrations but also the combination of harmonics due to the interaction of rotational frequency and its harmonics with the constant excitation frequency and its harmonics. Then the same results of coupling measurements and appearance of combination harmonics have been observed for slant crack in rotor: coupled longitudinal, bending and torsional vibrations [23, 24].

In practice the material parameters and the unbalance excitation force in the rotor systems are subjected to inherent physical variability and so are not exactly known. The dynamic properties of the rotor system can be deeply affected by small variations of some of these parameters and so they can be within a band of possible values due to uncertainties of some parameters such as stiffness and unbalance. However, the efficiency of the most practical indicators for health monitoring purposes is not analyzed in the presence of uncertainties present on rotating systems. The paper addresses this important issue of the changes in response of a cracked rotor under uncertain parameters and the robustness of indicators for monitoring rotating machinery.

Numerous methods have been developed to quantify physical uncertainties in a variety of computational problems e.g. the Monte Carlo approach, the perturbation method and the polynomial chaos expansions. The Monte Carlo simulations consists in including the uncertainties by calculating $\mathrm{n}$ times the deterministic problem, that is, for $\mathrm{n}$ independent samples of the random parameter. Therefore, the statistical response is easily obtained. With its easy use, the Monte Carlo method is one of the most frequently used. However, the convergence rate of this method is very slow which brings computations to 
be intensive really fast and not adapted to high-dimensional deterministic problems or high CPU time deterministic computations. In particular, rotordynamic problems are quite complex in a deterministic sense. The second type of methods includes either the perturbation method [25] random quantities in expansion of Taylor series or the Neumann method based on Neumann series expansion [26,27]. These methods obtain acceptable results only if the random fluctuations are small, therefore if we consider a problem with one excitation close to the resonance frequency, they can not be efficiently used. Finally, the polynomial chaos expansion associated with a Galerkin projection, named the Stochastic Finite Element Method [28] showed its effectiveness for quantifying uncertainties through the value of stochastic processes or variables in orthogonal bases of random variables. It is based on the homogeneous chaos theory of Wiener [29] and the original polynomial chaos expansion [28] that consists in a mean-square convergent expansion of multidimensional Hermite polynomials of normalized Gaussian variables. The Hermite polynomials are orthogonal with respect to the Gaussian measure then optimal exponential convergence is obtained for Gaussian inputs [30]. Besides, many extensions were made on the nature of the expansion (e.g. non Gaussian variables) to solve very different types of problems, especially in structural dynamics [31].

In this paper, due to the fact that determining all the Fourier components of the rotor system for the deterministic problem is already computationally intensive, the computational costs of Monte Carlo simulations can become impractical. Consequently, we use the polynomial chaos expansion method in the form of the stochastic finite element model that seems to be well adapted for a combination with the Harmonic Balance Method: the response of the Fourier components of the rotor system returns as random and is then expanded in the polynomial chaos basis. To validate the method, all the calculations are compared to those obtained from the combination of the Harmonic Balance Method and the Monte Carlo simulations.

First of all, a brief introduction of the rotor system and the modeling of the crack based on the notion of stiffness reduction are described. Then, the paper presents the numerical procedure for calculating the response of a cracked rotor under uncertain parameters. To quantify the effects of uncertainties on response variability in cracked rotor systems the stochastic response via the combination of the Harmonic Balance Method and the Polynomial Chaos Expansion procedure are explained and studied. Finally, the results obtained by applying the alternative approach via the Harmonic Balance Method and the Polynomial Chaos Expansion are compared with those evaluated by the Harmonic Balance Method with the Monte Carlo Simulations. The efficiency and the robustness of the combination of the Harmonic Balance Method with the Polynomial Chaos Expansion is demonstrated through different numerical simulations in order to analyze the random non-linear response of a cracked rotor against uncertain parameters and random excitation to assess its accuracy and the computation time.

\section{The model of the cracked rotor}

The layout of the cracked rotor system under consideration is shown in Figure 1. The rotor is composed of a shaft of length $0.5 \mathrm{~m}$ with one disc at the mid-span. All the values of the material properties and dimensions of the rotor are given in Table 1.

The uncraked rotor is discretized into Timoshenko beam finite elements, with four degrees of freedoms at each node, the axial and torsional degrees of freedom being not considered. After assembling the various shaft elements, the rigid disc and the bearing supports, the equations of the uncracked rotor can be written as

$$
\mathbf{M} \ddot{\mathbf{x}}+\mathbf{D} \dot{\mathbf{x}}+\mathbf{K x}=\mathbf{f}+\mathbf{q}
$$


where $\mathbf{f}$ and $\mathbf{q}$ contain the gravitational and balance forces, respectively. The mass matrix $\mathbf{M}$ includes the mass matrices of the shaft and the rigid disc. The matrix $\mathbf{D}$ combines the effects of the shaft's internal damping, damping of the supports, and gyroscopic moments. The matrix $\mathbf{K}$ includes the stiffness matrices of the shaft and the supports, together with the circulatory matrix which accounts for the shaft's internal damping.
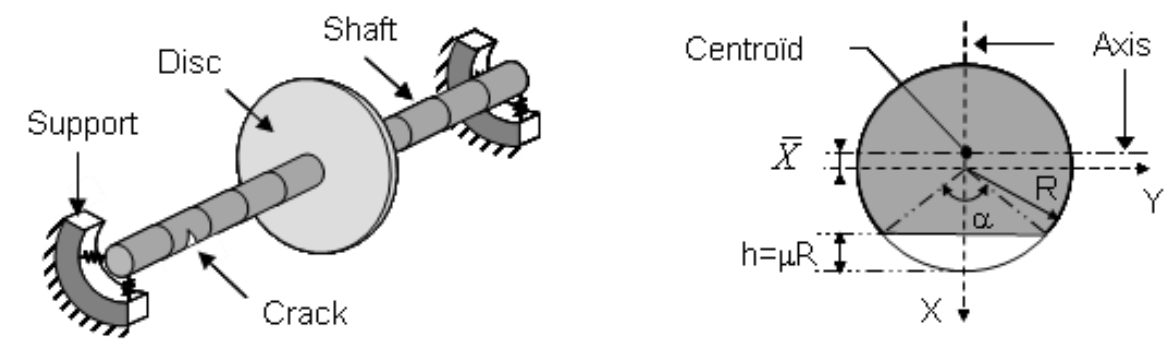

Figure 1: Finite-element model of the rotor and the cracked-beam section

\begin{tabular}{ccc}
\hline Notation & Description & Value \\
\hline$R$ & radius of the rotor shaft & $0.005 \mathrm{~m}$ \\
$L$ & length of the rotor shaft & $0.5 \mathrm{~m}$ \\
$R_{D}$ & outer radius of the disk & $0.025 \mathrm{~m}$ \\
$h_{D}$ & thickness of the disk & $0.015 \mathrm{~m}$ \\
$E$ & Young's modulus of elasticity & $2.110^{11} \mathrm{~N} \cdot \mathrm{m}^{-2}$ \\
$G$ & shear modulus & $7.710^{10} \mathrm{~N} \cdot \mathrm{m}^{-2}$ \\
$\rho$ & density & $7800 \mathrm{~kg} \cdot \mathrm{m}^{-3}$ \\
$\nu$ & Poisson ratio & 0.3 \\
$\eta$ & coefficient of damping & $0.110^{-5}$ \\
$K_{s}$ & stiffness of supports & $210^{6} \mathrm{~N} \cdot \mathrm{m}^{-1}$ \\
\hline
\end{tabular}

Table 1: Value of the physical parameters

Then, due to strain energy concentration in the vicinity of the tip of the crack under load, the presence of a transverse crack introduces local flexibility. For a comprehensive literature survey of various crack modeling techniques, see $[2,3]$.

In this paper, the model proposed by Mayes and Davies [4,5] is used in order to locally represent the stiffness properties of the crack cross section. This model considers the reduction of the second moment of area $\Delta I$ of the element at the location of the crack that may be defined by

$$
\Delta I=I_{0}\left(\frac{\frac{R}{l}\left(1-\nu^{2}\right) F(\mu)}{1+\frac{R}{l}\left(1-\nu^{2}\right) F(\mu)}\right),
$$

where $I_{0}, R, l$, and $\nu$ are the second moments of area, beam radius, length of the section and Poisson's ratio, respectively. $\mu$ is the non-dimensional crack depth and is given by $\mu=\frac{h}{R}$ where $h$ defines the 
crack depth of the beam, as shown in Figure 1. $F(\mu)$ defines the non-linear compliance as a function of variations in non-dimensional crack depth $\mu$, which can be derived from a series of experiments using chordal cracks (see Mayes and Davies [4,5]). For the interested reader, the complete expressions of the stiffness matrix $\mathbf{K}_{\text {crack }}$ at the transverse crack location are given in [17]. In this study, the crack model is restricted to a chordal crack.

Then, one of the models of a crack is that of Mayes and Davies [5] where the opening and closing of the crack were described by a cosine function by assuming that the gravity force is much greater than the imbalance force: when a cracked rotor rotates slowly under the load of its own weight, the crack will open and close once per revolution. This periodic opening and closing of the crack is called "breathing" phenomenon [1]. The function describing a simple crack breathing phenomenon such as discussed by Sinou and Lees $[17,32]$, typical in weight dominated systems, may be approximated by a cosine function $g(t)$

$$
g(t)=\frac{1-\cos \omega t}{2}
$$

where $\omega$ defines the rotational speed of the rotor. If $g(t)=0$, the crack is closed and has no effect on the dynamic behaviour of the rotor (i.e. the rotor may be treated as uncracked). If $g(t)=1$, the crack is fully open.

Finally, the equations of the cracked rotor can be written as

$$
\mathbf{M} \ddot{\mathbf{x}}+\mathbf{D} \dot{\mathbf{x}}+\left(\mathbf{K}-g(t) \mathbf{K}_{c}\right) \mathbf{x}=\mathbf{f}+\mathbf{q}
$$

where $\ddot{\mathbf{x}}, \dot{\mathbf{x}}$ and $\mathbf{x}$ are the acceleration, velocity and displacement vectors. $\mathbf{M}$ and $\mathbf{K}$ are the mass and stiffness matrices of the complete uncracked rotor. $\mathbf{f}$ and $\mathbf{q}$ are the gravitational and balance forces, respectively. The global stiffness matrix $\mathbf{K}_{c}$ due to the presence of the crack is given by

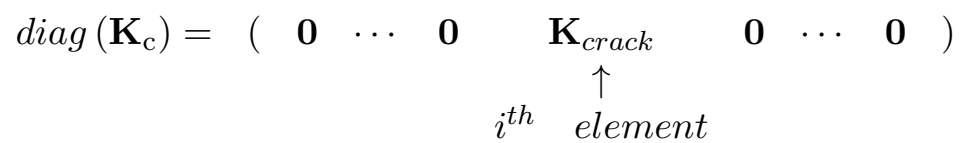

where $\mathbf{K}_{\text {crack }}$ defines the stiffness matrix of the crack element. It is situated at the $i^{\text {th }}$ beam location. $\mathbf{0}$ defines the $8 \times 8$ null matrix.

\section{Response of the cracked rotor under uncertain parameters via the Harmonic Balance Method and the Polynomial Chaos Expansion (HBM-PCE)}

\subsection{Deterministic response via the Harmonic balance Method}

As previously indicated, the above equations of the cracked rotor have a time-dependent coefficient due to the fact that the crack breathes when the system rotates. The amount of open part of the crack constantly varies with the rotation of the shaft, thereby changing the stiffness of the cracked rotor. The global stiffness matrix of the rotor consists of a constant part $\mathbf{K}$ and a time dependent part $g(t) \mathbf{K}_{c}$.

In order to determine the periodic solutions of the cracked rotor system, we assume that the dynamical responses of the cracked rotor system can be approximated by a truncated Fourier series of 
order $m$ with a fundamental frequency

$$
\mathbf{x}(t)=\mathbf{A}_{0}+\sum_{k=1}^{m}\left(\mathbf{A}_{k} \cos (k \omega t)+\mathbf{B}_{k} \sin (k \omega t)\right)
$$

where $\omega$ defines the rotational speed of the cracked rotor system. $\mathbf{A}_{0}, \mathbf{A}_{k}$ and $\mathbf{B}_{k}$ (with $k=1, \cdots, m$ ) define the unknown coefficients of the finite Fourier series. The number of harmonic coefficients $m$ is selected on the basis of the number of significant harmonics expected in the dynamical response of the crack rotor system.

Then, it may be observed that the gravitational force $\mathbf{f}$ and the unbalance force $\mathbf{q}$ can also be exactly defined by finite Fourier series (with constant components and first-order periodic components in the frequency domain, respectively). Expressions are given by

$$
\begin{gathered}
\mathbf{f}=\mathbf{C}_{0}^{f} \\
\mathbf{q}=\mathbf{C}_{1}^{q} \cos (\omega t)+\mathbf{S}_{1}^{q} \sin (\omega t)
\end{gathered}
$$

where $\mathbf{C}_{0}^{f}$ is the vector of the constant Fourier components of the gravitational force. $\mathbf{C}_{1}^{q}$ and $\mathbf{S}_{1}^{q}$ are first-order periodic components in the frequency domain of the unbalance force.

By combining the approximated dynamical response of the rotor $\mathbf{x}$ (see equation 6 ) and the the global mechanism of the time dependent part (see equation 3), the expression $g(t) \mathbf{K}_{c} \mathbf{x}$ can be approximated by considering truncated Fourier series of order $m$

$g(t) \mathbf{K}_{c} \mathbf{x}=\sum_{k=0}^{m} \mathbf{K}_{c}\left(-\frac{1}{2} \mathbf{A}_{k-1}+\frac{1}{2} \mathbf{A}_{k}-\frac{1}{4} \mathbf{A}_{k+1}\right) \cos (k \omega t)+\sum_{k=1}^{m} \mathbf{K}_{c}\left(-\frac{1}{2} \mathbf{B}_{k-1}+\frac{1}{2} \mathbf{B}_{k}-\frac{1}{4} \mathbf{B}_{k+1}\right) \sin (k \omega t)$

with $\mathbf{B}_{0}=\mathbf{A}_{-1}=\mathbf{0}$ and $k+1<m$. Finally, substituting Equations 6, 7, 8, 9 into Equations 4 yields a set of $(2 m+1) \times n$ linear equations (where $n$ is the number of degrees of freedom for the complete cracked rotor)

$$
\left(\boldsymbol{\Lambda}+\boldsymbol{\Lambda}_{c}\right) \boldsymbol{\Theta}=\boldsymbol{\Gamma}
$$

with

$$
\begin{aligned}
& \boldsymbol{\Theta}=\left[\begin{array}{lllllllllll}
\mathbf{A}_{0} & \mathbf{A}_{1} & \mathbf{B}_{1} & \mathbf{A}_{2} & \mathbf{B}_{2} & \cdots & \mathbf{A}_{k} & \mathbf{B}_{k} & \cdots & \mathbf{A}_{m} & \mathbf{B}_{m}
\end{array}\right]^{T} \\
& \boldsymbol{\Gamma}=\left[\begin{array}{lllllllllll}
\mathbf{C}_{0}^{f} & \mathbf{C}_{1}^{q} & \mathbf{S}_{1}^{q} & \mathbf{0} & \mathbf{0} & \cdots & \mathbf{0} & \mathbf{0} & \cdots & \mathbf{0} & \mathbf{0}
\end{array}\right]^{T}
\end{aligned}
$$

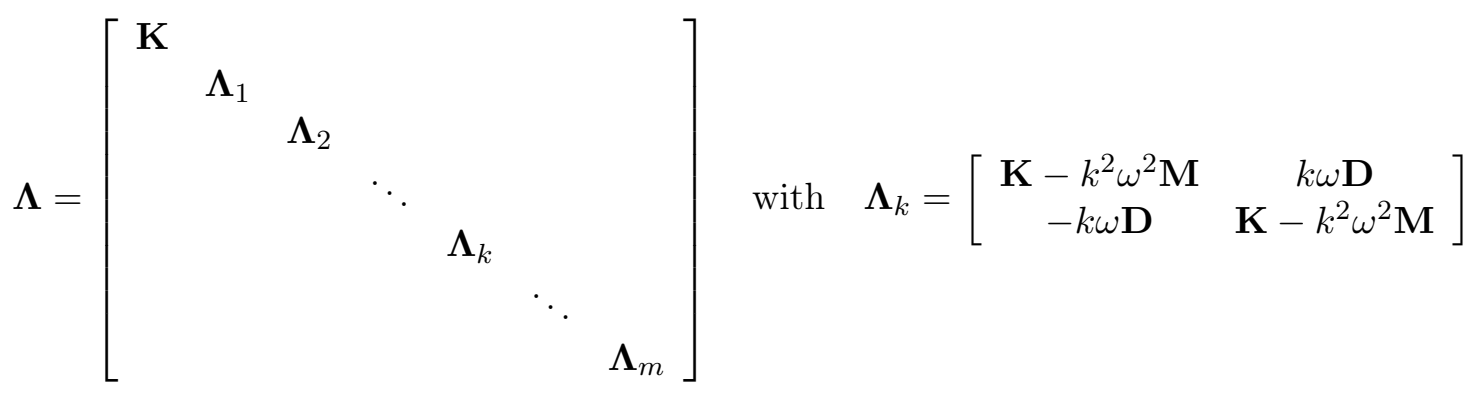




$$
\boldsymbol{\Lambda}_{c}=\frac{1}{4}\left[\begin{array}{cccccccccccc}
2 \mathbf{K}_{c} & -\mathbf{K}_{c} & & & & & & & & & \\
-2 \mathbf{K}_{c} & 2 \mathbf{K}_{c} & \mathbf{0} & -\mathbf{K}_{c} & \mathbf{0} & & & & & & \\
\mathbf{0} & \mathbf{0} & 2 \mathbf{K}_{c} & \mathbf{0} & -\mathbf{K}_{c} & & & & & & \\
& -\mathbf{K}_{c} & \mathbf{0} & 2 \mathbf{K}_{c} & \mathbf{0} & -\mathbf{K}_{c} & \mathbf{0} & & & & \\
& \mathbf{0} & -\mathbf{K}_{c} & \mathbf{0} & 2 \mathbf{K}_{c} & \mathbf{0} & -\mathbf{K}_{c} & & & & \\
& & & & & \ddots & & & & & \\
& & & & -\mathbf{K}_{c} & \mathbf{0} & 2 \mathbf{K}_{c} & \mathbf{0} & -\mathbf{K}_{c} & \mathbf{0} & \\
& & & & \mathbf{0} & -\mathbf{K}_{c} & \mathbf{0} & 2 \mathbf{K}_{c} & -\mathbf{0} & -\mathbf{K}_{c} & \\
& & & & & & & & \ddots & & \\
& & & & & & & -\mathbf{K}_{c} & \mathbf{0} & 2 \mathbf{K}_{c} & \mathbf{0} \\
& & & & & & & \mathbf{0} & -\mathbf{K}_{c} & \mathbf{0} & 2 \mathbf{K}_{c}
\end{array}\right]
$$

The unknown Fourier coefficients $\boldsymbol{\Theta}$ can be found solving Equation 10.

\subsection{Stochastic response via the Harmonic Balance Method-Polynomial Chaos Ex- pansion (HBM-PCE)}

Uncertainties on the stiffness parameters and on the unbalance excitation force have to be modeled. $\boldsymbol{\Lambda}, \boldsymbol{\Theta}$ and $\boldsymbol{\Gamma}_{u}$ are random processes with argument $\tau$ denoting the random character, $\boldsymbol{\Lambda}_{c}$ is considered as deterministic. Equation 10 can then be rewritten in a random way such that

$$
\left(\boldsymbol{\Lambda}(\tau)+\boldsymbol{\Lambda}_{c}\right) \boldsymbol{\Theta}(\tau)=\boldsymbol{\Gamma}(\tau)
$$

In fact, excitation force $\boldsymbol{\Gamma}$ consists of a deterministic part that is the gravity component $\boldsymbol{\Gamma}_{g}$ and of a random part that is the unbalance component $\boldsymbol{\Gamma}_{u}(\tau)$ such as

$$
\boldsymbol{\Gamma}=\boldsymbol{\Gamma}_{g}+\boldsymbol{\Gamma}_{u}(\tau)
$$

From Equation 12, it yields :

$$
\boldsymbol{\Gamma}_{g}=\left[\begin{array}{llll}
\mathbf{C}_{0}^{f} & \mathbf{0} & \cdots & \mathbf{0}
\end{array}\right]^{T} \quad \text { and } \quad \boldsymbol{\Gamma}_{u}(\tau)=\left[\begin{array}{llllll}
\mathbf{0} & \mathbf{C}_{1}^{q}(\tau) & \mathbf{S}_{1}^{q}(\tau) & \mathbf{0} & \cdots & \mathbf{0}
\end{array}\right]^{T}
$$

$\boldsymbol{\Lambda}$, defined by its deterministic expression by Equation 13, can be randomized using the Karhunen Loeve expansion with a Gaussian law on the stiffness of the rotor. The use of Karhunen-Loeve expansion allows to work with random variables instead of random processes $[28,33]$. It should be noted that the support boundary conditions are taken as deterministic so the stiffness associated and denoted $\mathbf{K}_{b}$ is not taken as random. Then, considering that $\mathbf{K}=\mathbf{K}_{b}+\widetilde{\mathbf{K}}$, Equation 13 becomes

$$
\Lambda=\Lambda_{d}+\Lambda_{r}(\tau)
$$

where $\boldsymbol{\Lambda}_{d}$ is the deterministic part of $\boldsymbol{\Lambda}$ defined by

$$
\boldsymbol{\Lambda}_{d}=\left[\begin{array}{cccc}
\mathbf{K}_{b} & & & \\
& \boldsymbol{\Lambda}_{1}^{d} & & \\
& & \ddots & \\
& & & \boldsymbol{\Lambda}_{m}^{d}
\end{array}\right] \quad \text { with } \quad \boldsymbol{\Lambda}_{k}^{d}=\left[\begin{array}{cc}
\mathbf{K}_{b}-k^{2} \omega^{2} \mathbf{M} & k \omega \mathbf{D} \\
-k \omega \mathbf{D} & \mathbf{K}_{b}-k^{2} \omega^{2} \mathbf{M}
\end{array}\right]
$$


and $\boldsymbol{\Lambda}_{r}(\tau)$ is the random one given by

$$
\mathbf{\Lambda}_{r}(\tau)=\left[\begin{array}{lll}
\widetilde{\mathbf{K}}(\tau) & & \\
& \ddots & \\
& & \widetilde{\mathbf{K}}(\tau)
\end{array}\right]
$$

$\widetilde{\mathbf{K}}(\tau)$ is expanded using the Karhunen-Loeve expression implemented in the Galerkin formulation of the finite element method [28] such as

$$
\widetilde{\mathbf{K}}(\tau)=\sum_{i=1}^{L} \overline{\widetilde{\mathbf{K}}}_{i}\left(1+\delta_{i}^{K} \xi_{i}^{K}(\tau)\right)
$$

in which $\left\{\xi_{i}\right\}, i=1$ to $L$, is a set of orthonormal random variables, $\overline{\mathbf{Z}}$ is the mean of random quantity $\mathbf{Z}$ and $\delta^{Z}$ is its variation coefficient.

Considering the unbalance excitation force $\boldsymbol{\Gamma}_{u}(\tau)$, several random ways of modeling can be possible. One simple one but sufficiently representative of the reality is done through the Polynomial Chaos Expansion with a Gaussian law. The associated basis is an orthogonal basis set of random variables, represented in a mean-square convergent expansion in terms of multidimensional Hermite polynomials of normalized Gaussian variables [28]. We have

$$
\boldsymbol{\Gamma}_{u}(\tau)=\sum_{j=0}^{\infty} \mathbf{F}_{u_{j}} \Psi_{j}(\underline{\xi}(\tau))
$$

The unknown solution $\Theta(\tau)$ is also then expanded in chaos polynomials such as

$$
\boldsymbol{\Theta}(\tau)=\sum_{j=0}^{\infty} \mathbf{Y}_{j} \Psi_{j}(\underline{\xi}(\tau))
$$

where $\Psi_{j}(\underline{\xi}(\tau))$ refers to a rearrangement of the p-order finite dimensional orthogonal polynomials with respect to the Gaussian function which constitute a complete basis in the space of second-order random variables ; $\mathbf{Y}_{j}$ is the unknown deterministic $j^{\text {th }}$ vector associated with $\Psi_{j}(\underline{\xi}(\tau))$ and $\underline{\xi}=\left\{\xi_{r}\right\}$, set of orthonormal random variables [28]. Finally, after projection on the chaos polynomial basis, the stochastic system to solve is written as

$$
\mathbf{A Y}=\mathbf{F}
$$

where each component $[\mathbf{A}]_{i j}$ of $\mathbf{A}$ is

$$
[\mathbf{A}]_{i j}=\left\{\left[\boldsymbol{\Lambda}_{c}+\boldsymbol{\Lambda}_{d}+\sum_{k=1}^{L} \overline{\boldsymbol{\Lambda}}_{r k}\right]\left\langle\Psi_{i}^{2}\right\rangle \delta_{i j}+\sum_{k=1}^{L} \overline{\boldsymbol{\Lambda}}_{r k} \delta_{k}^{K} c_{i j k}\right\}
$$

$\langle Z\rangle$ corresponds to the mathematical expectation of quantity $Z, \delta_{i j}$ is the Kronecker symbol and $c_{i j k}{ }^{K}$ is defined by $[28]$

$$
c_{i j k^{K}}=\left\langle\xi_{k}^{K} \Psi_{i} \Psi_{j}\right\rangle
$$

Finally, vector $\mathbf{F}(\tau)$ of forces projected on the polynomial chaos basis has polynomial chaos vector terms given by

$$
\mathbf{F}_{j}(\tau)=\boldsymbol{\Gamma}_{g} \delta_{0 j}\left\langle\Psi_{i}^{2}\right\rangle \delta_{i j}+\mathbf{F}_{u_{j}}\left\langle\Psi_{i}^{2}\right\rangle \delta_{i j}
$$




\section{Numerical results}

In this section, efficiency of the proposed methodology based on the combination of the Harmonic Balance Method with the Polynomial Chaos Expansion will be firstly demonstrated through numerical simulations of the rotor system with a breathing crack. Then, the random non-linear response of a cracked rotor against uncertain parameters and the effects of uncertainties from stiffness properties of the rotor and external forces will be analyzed in order to propose a robust identification of damage in rotors. The random aspect will be presented through the mean and the standard deviation of the response. The standard deviation $\sigma_{r}$ is defined by

$$
\sigma_{r}=\sqrt{\left\langle\left(\boldsymbol{\Theta}(\tau)-\boldsymbol{\Theta}_{0}\right)^{2}\right\rangle}
$$

Given the orthogonality of the polynomial chaoses, Equation 28 can be rewritten as

$$
\sigma_{r}=\sqrt{\sum_{j=1}^{\infty} \boldsymbol{\Theta}_{i}^{2}(\tau)\left\langle\Psi_{i}^{2}\right\rangle}
$$

\subsection{Choice of the terms in the expansions}

Clearly, in a computational context, the summations in the previous expressions are truncated after some term $P$. In particular, response $\Theta(\tau)$ given by Equation 23 can be written as

$$
\widetilde{\boldsymbol{\Theta}}(\tau)=\sum_{j=0}^{P} \mathbf{Y}_{j} \Psi_{j}(\underline{\xi}(\tau))
$$

where $P$, the total number of polynomial chaoses used in the expansion (excluding the $0^{\text {th }}$-order term), can be determined by $P=1+\sum_{s=1}^{p} \frac{1}{s !} \prod_{r=0}^{s-1}(L+r), p$ being the order of the homogeneous chaos being used [28]. $\left\{\Psi_{j}(\theta)\right\}_{j=0 \text { to } P}$ refers to a rearrangement of the $p^{\text {th }}$-order finite-dimension orthogonal polynomials with respect to the Gaussian function, which constitute a complete basis of the space of the second-order random variables.

Besides, the uncertainty is quantified identically along the stiffness of the rotor, consequently the random modeling of $\widetilde{\mathbf{K}}$ uses only one random variable. Thus, Equation 21 can be rewritten as

$$
\widetilde{\mathbf{K}}(\tau)=\overline{\widetilde{\mathbf{K}}}\left(1+\delta^{K} \xi^{K}(\tau)\right) .
$$

It should be noted that the normal distribution assumed has been truncated to avoid negative values of the parameter, since it is physically strictly positive.

Moreover, the expansion of unbalance force $\boldsymbol{\Gamma}_{u}$ can reasonably be truncated to order 1 which is equivalent to consider the Karhunen-Loeve expansion. Then Equation 22 can be reduced to

$$
\widetilde{\boldsymbol{\Gamma}}_{u}(\tau)=\overline{\widetilde{\boldsymbol{\Gamma}}}_{u}\left(1+\delta^{\Gamma_{u}} \xi^{\Gamma_{u}}(\tau)\right)
$$

considering one random variable. Integrating these hypotheses in Equation 24, the system of equations to solve becomes

$$
\widetilde{\mathbf{A}} \widetilde{\mathbf{Y}}=\widetilde{\mathbf{F}}
$$


where each component $[\widetilde{\mathbf{A}}]_{i j}$ of $\widetilde{\mathbf{A}}$ is

$$
[\widetilde{\mathbf{A}}]_{i j}=\left[\left(\boldsymbol{\Lambda}_{c}+\boldsymbol{\Lambda}_{d}+\overline{\boldsymbol{\Lambda}_{r}}\right)\left\langle\Psi_{i}^{2}\right\rangle \delta_{i j}+\overline{\boldsymbol{\Lambda}_{r}} \delta^{K} c_{i j 1^{K}}\right]
$$

and polynomial chaos vectors $\widetilde{\mathbf{F}}_{j}$ are given by

$$
\widetilde{\mathbf{F}}_{j}(\tau)=\boldsymbol{\Gamma}_{g} \delta_{0 j}\left\langle\Psi_{i}^{2}\right\rangle \delta_{i j}+\overline{\widetilde{\mathbf{F}}}_{u_{j}}\left[\left\langle\Psi_{i}^{2}\right\rangle \delta_{0 j} \delta_{i j}+\delta^{\Gamma_{u}}\left\langle\Psi_{i}(\underline{\xi}) \xi^{\Gamma_{u}}\right\rangle\right]
$$

where $\xi=\left\{\xi^{K}, \xi^{\Gamma_{u}}\right\}$.

Finally, the standard deviation is also built from a truncated expansion that is

$$
\sigma_{r}=\sqrt{\sum_{j=1}^{P} \boldsymbol{\Theta}_{i}^{2}(\tau)\left\langle\Psi_{i}^{2}\right\rangle} / \boldsymbol{\Theta}_{0}
$$

It should be noted that the size of the system of equations depends on $P$ and is obviously higher than those of the deterministic problem.

\subsection{Comparison between Monte-Carlo Simulations and Polynomial Chaos Expan- sion}

The example of the non-linear vertical response at the middle of the cracked rotor, for one nondimensional crack depth $\mu$ equal to 1 and with variations for both shaft stiffness of the rotor system and the excitation forces of $2 \%$ is taken to show the efficiency and the robustness of the HBM-PCE method versus the HBM-MCS method. The reference solution is obtained with the HBM-MCS method using 5000 samples. We present the results from the HBM-PCE method using several orders in the expansion : $p=5,10$ and 20 (for listing of coefficients, see [28]). Here, for a better legibility, the mean (Figure 2) and the standard deviation (Figure 3) only for harmonic 2 of the random response are presented. It should be noted that similar conclusions were observed for the other harmonics of the response. Figures 2 and 3 present each : (a) the response on the whole frequency range studied and (b) a zoom for example on the resonance second peak. Clearly, for both mean and standard deviation, we can observe that, outside the resonances, the HBM-PCE solution is identical to that obtained by the MCS whatever the order $p$. The more important area where differences between the HBM-PCE solution and the the HBM-MCS is located at each sub-critical speed. Figures 4 and 5 show the mean and the standard deviation respectively for the four harmonics of the non-linear vertical response computed from both methods. Here again, the results for the random response are very close between both methods even around the resonances. These variations are only due the very small damping taken into account in the present study. For the interested reader, Figures 6 illustrated the non-linear vertical response with a higher damping (i.e. $\eta=10^{-5}$ ). It clearly demonstrated that the difference between HBM-PCE and HBM-MCS solutions decreases with the value of the damping as also mentioned in PhD thesis of Dessombz [34]. However, it is clearly seen that the HBM-PCE gets better with $p$, even if the damping is very small (i.e. $\eta=0.110^{-5}$ ). Besides, we can stop to order 20 since it seems to be sufficient to represent correctly the random behavior of the response of the cracked rotor due to the uncertain inputs. 


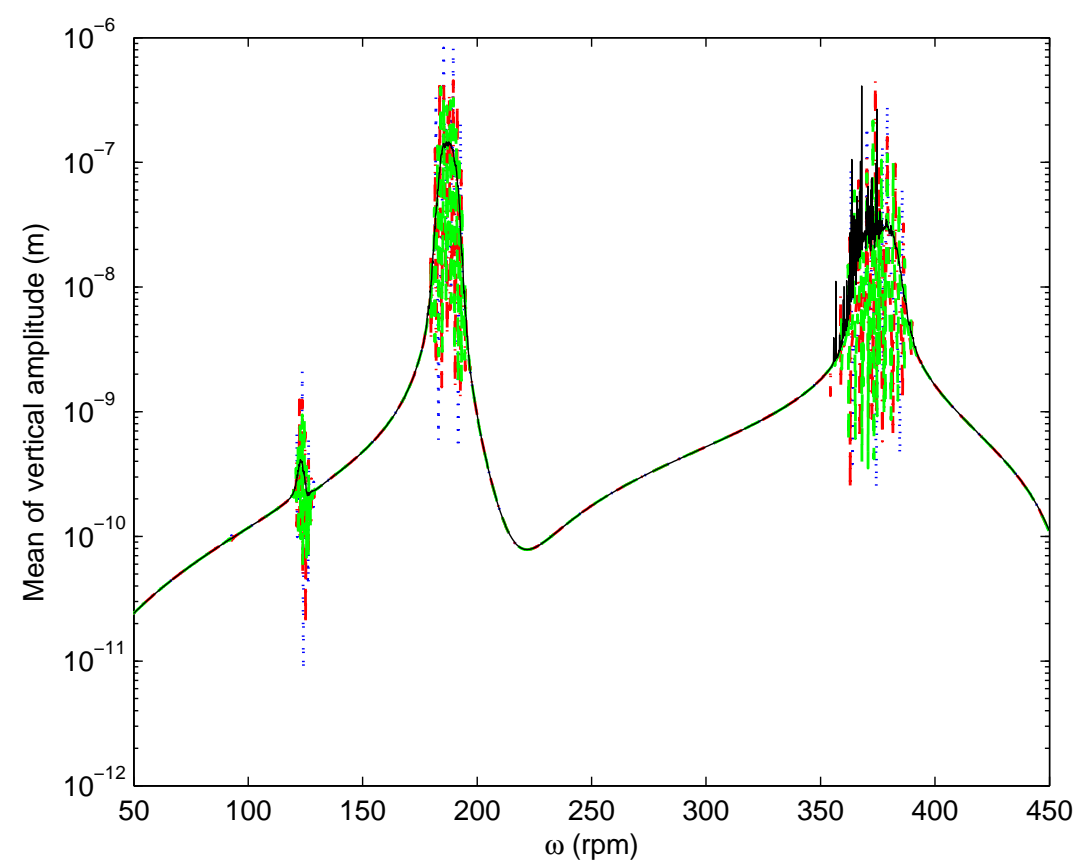

(a)

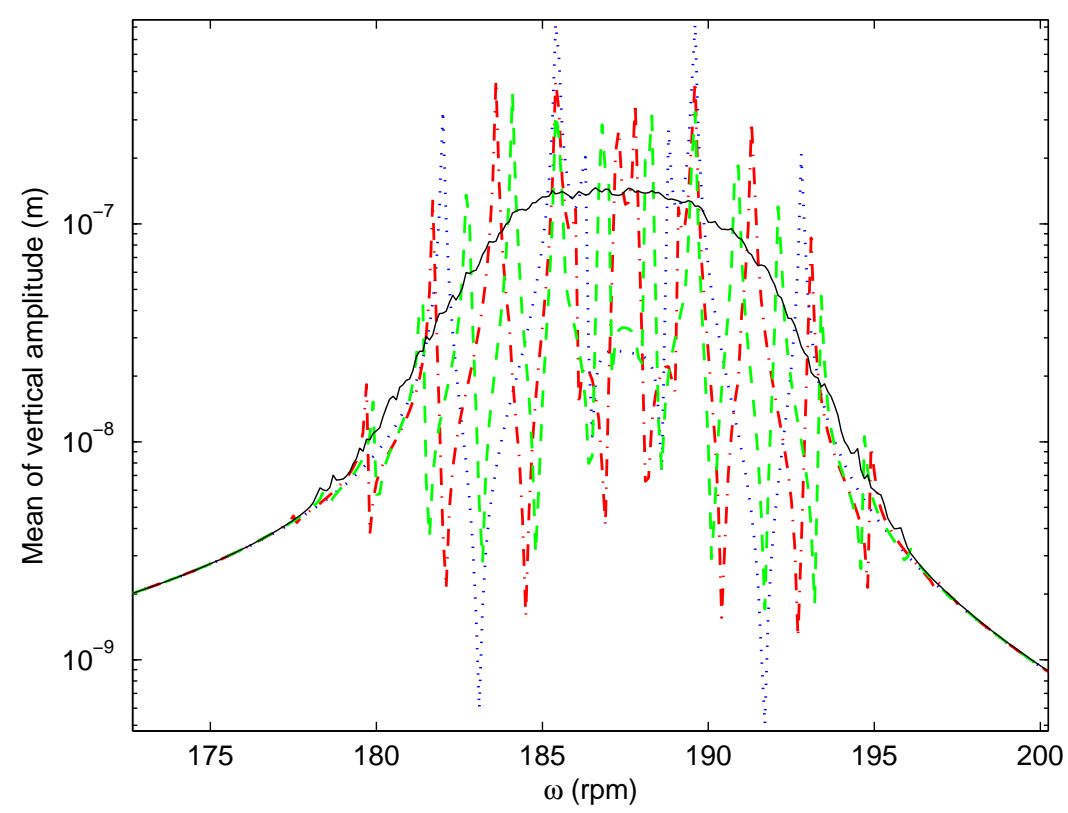

(b)

Figure 2: Mean of the second harmonic order computed by the MCS (solid line) and by the PCE of several orders : order 5 (dotted lines), order 10 (dotted-dashed lines), order 20 (dashed lines). (a) : whole frequency range, (b) : zoom on the second peak of resonance 


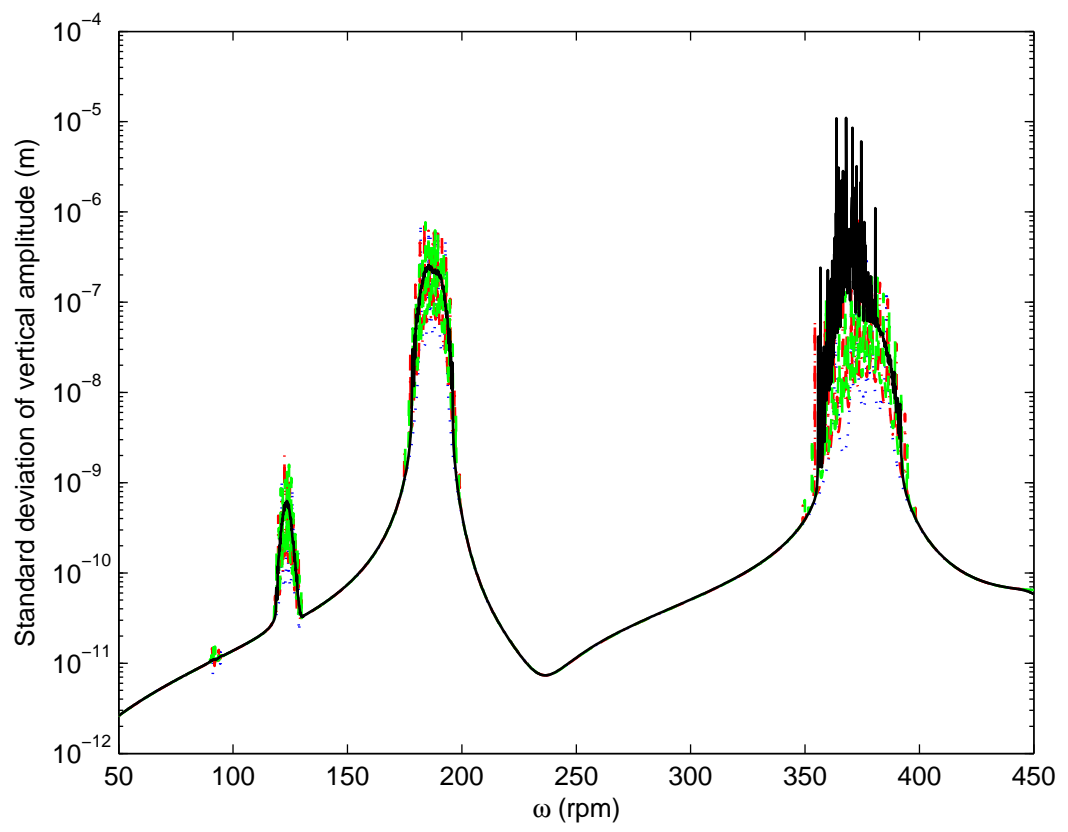

(a)

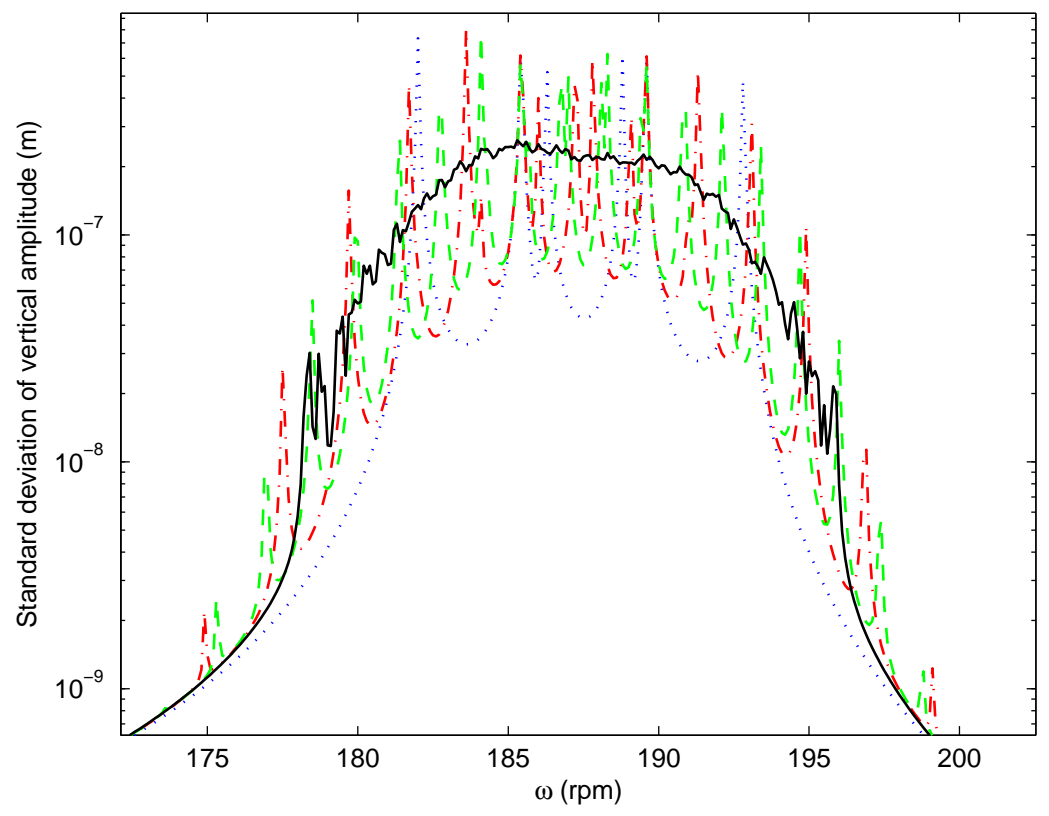

(b)

Figure 3: Standard deviation of the second harmonic order computed by the MCS (solid line) and by the PCE of several orders : order 5 (dotted lines), order 10 (dotted-dashed lines), order 20 (dashed lines). (a) : whole frequency range, (b) : zoom on the second peak of resonance 


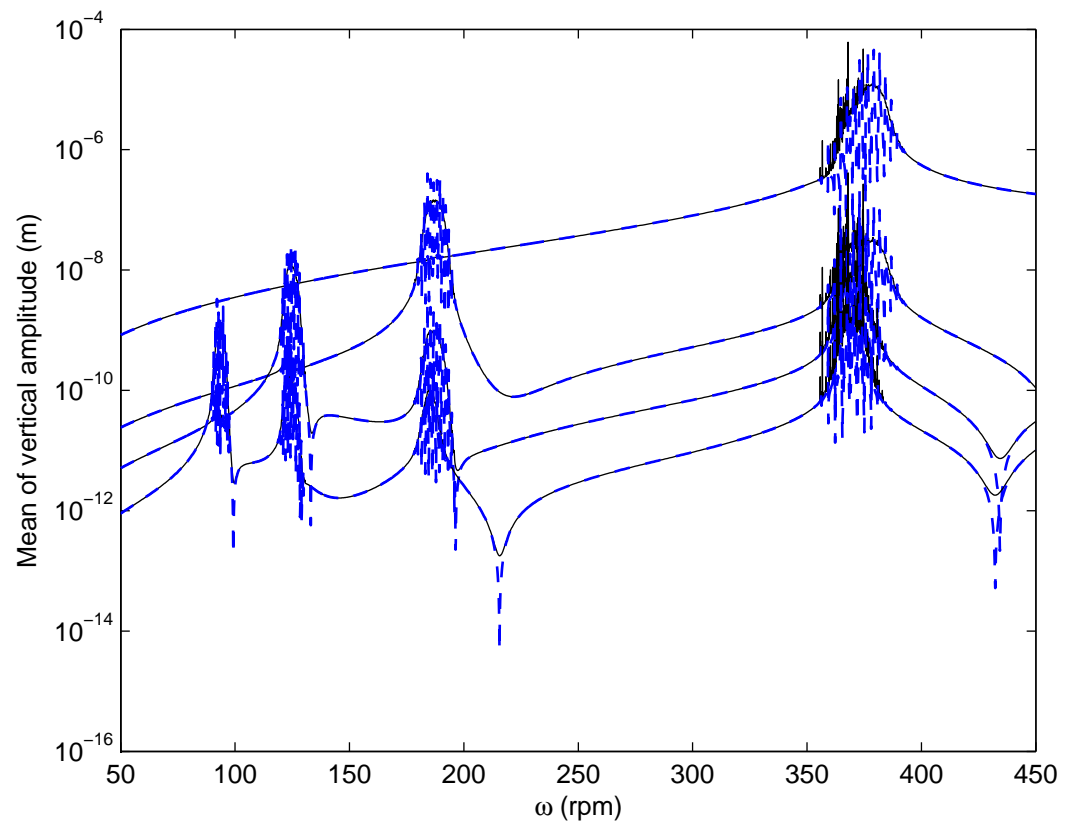

Figure 4: Mean of the non-linear response. Comparison between the MCS method (solid line) and the PCE method with order 20 (dashed lines) for the four harmonics of the response : top (harmonic 1) to the bottom (harmonic 4) 


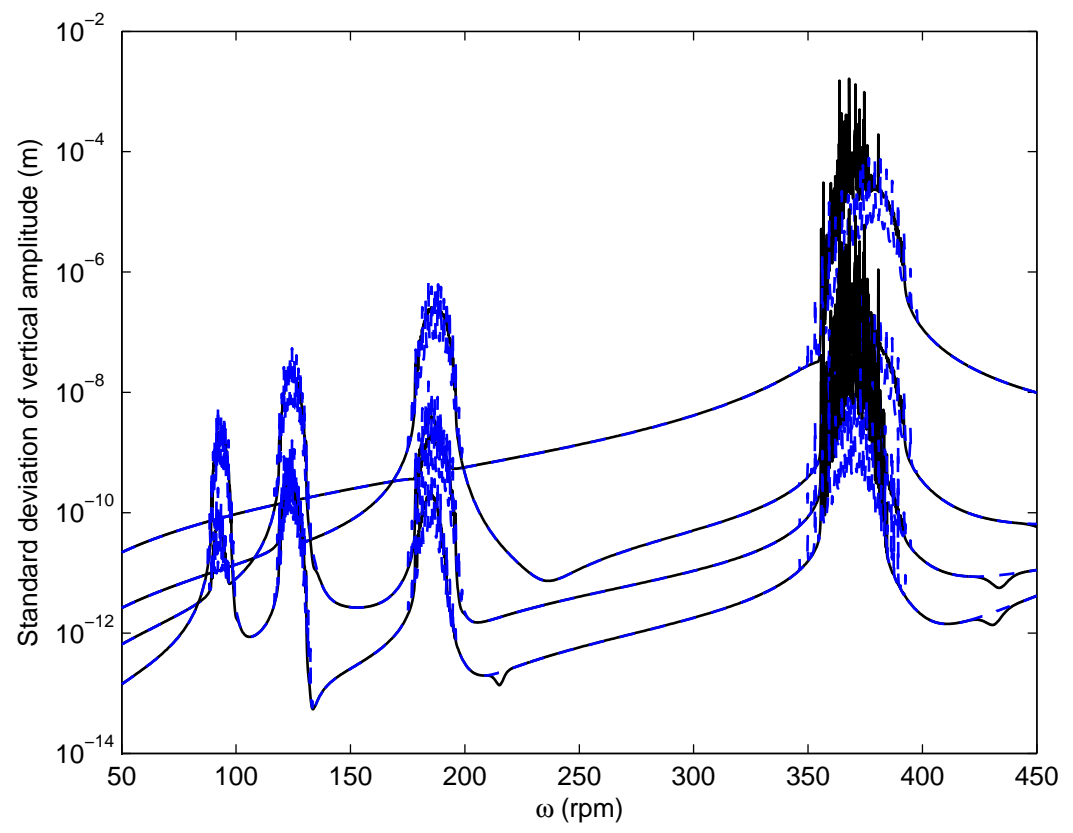

Figure 5: Standard deviation of the non-linear response. Comparison between the MCS method (solid line) and the PCE method with order 20 (dashed lines) for the four harmonics of the response : top (harmonic 1) to the bottom (harmonic 4) 


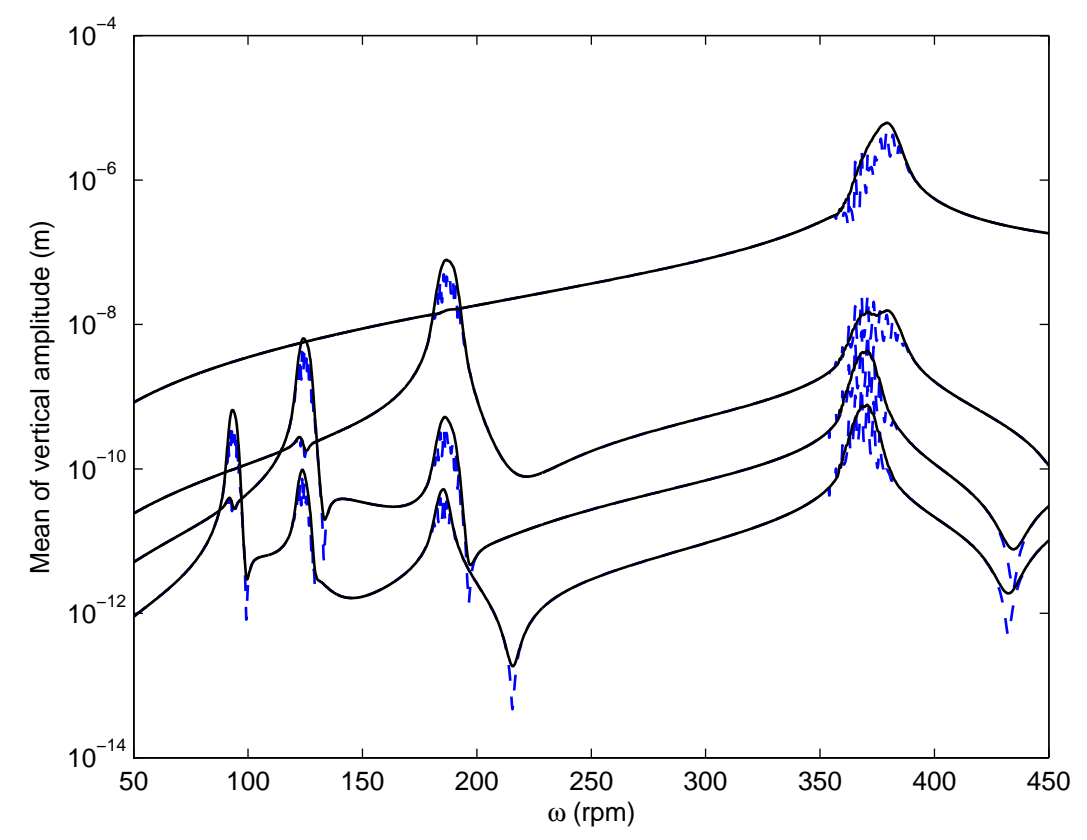

(a)

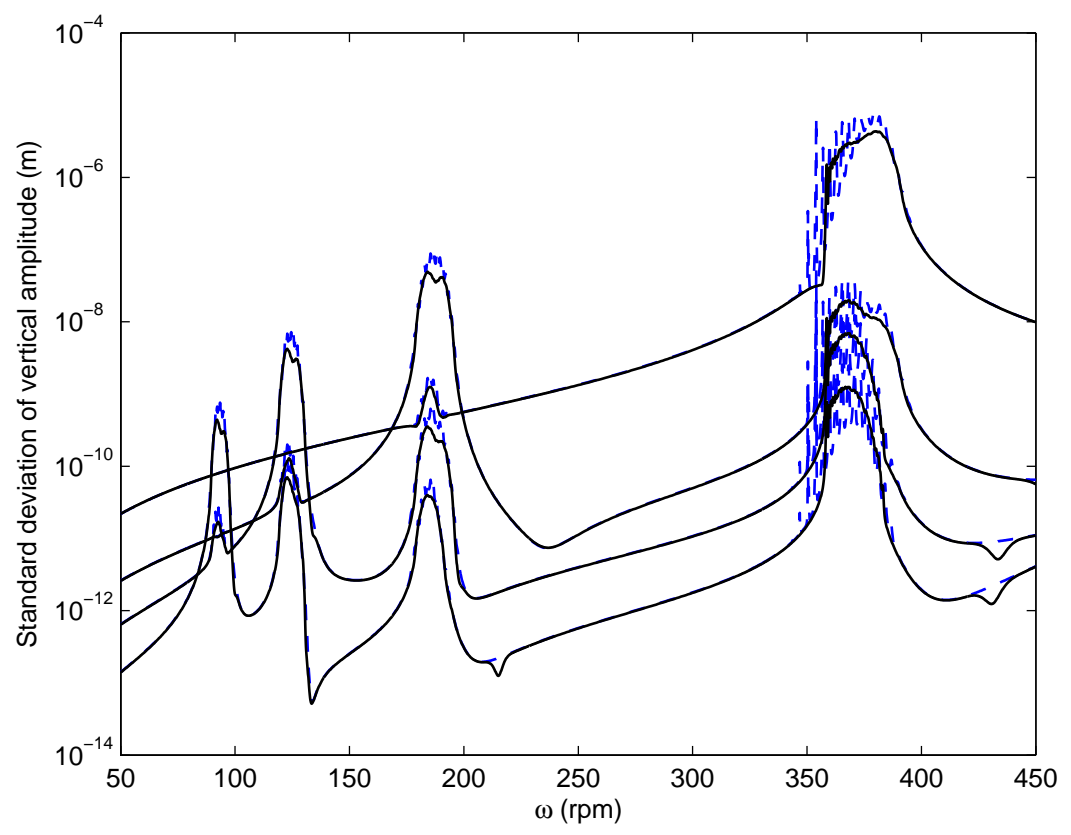

(b)

Figure 6: Mean and standard deviation of the vertical non-linear response for $\eta=10^{-5}$. Comparison between the MCS method (solid line) and the PCE method with order 20 (dashed lines) for the four harmonics of the response : top (harmonic 1) to the bottom (harmonic 4). (a) : Mean, (b) : Standard deviation 


\subsection{Efficiency of the crack detection based on the non-linear responses with un- certainties}

In this first part of the parametric studies, the non-dimensional crack depth $\mu$ is equal to 1 (corresponding to the loss of half the shaft's area). We consider random variations for the stiffness properties of shaft of the rotor system (applied actually on the Young modulus) and for the excitation forces. Three cases are investigated in which the coefficients of variation of the shaft's stiffness and of the excitation forces are taken equal to $1 \%, 2 \%$ and $5 \%$ respectively. We recall that in this case the cost of calculation may be high since it is directly linked to the number of polynomials and consequently to the order of polynomial chaoses and the number of random parameters.

Figures 7 show the mean value and the standard deviation of the non-linear vertical responses at the middle of the cracked rotor for these three cases. First of all, it appears that variations of the mean value and of the standard deviation increase when the rotor is passing through $\frac{1}{n}$ sub-critical speeds. For example, the $1 \times$ vertical response of the cracked rotor generates highly prominent amplitudes when the running speed is at the first critical speed and approximately $\frac{1}{2}$ of the first critical speed. An increase of the $2 \times$ vertical response at the critical speed, $\frac{1}{2}$ and $\frac{1}{3}$ of the first critical speed can also be observed. Finally, the $3 \times$ and $4 \times$ vertical responses increase at the critical speed, $\frac{1}{2}, \frac{1}{3}$ and $\frac{1}{4}$ of the first critical speed. However it is clear that only $1 \times$ component is dominant at first critical speed and $2 \times$ component is very small. Similarly, $2 \times$ component is very small while $2 \times$ component is very dominant at $\frac{1}{2}$ of critical speed. So, increases of the $n \times$ amplitudes at $\frac{1}{m}$ of the first critical speed (with $m \leq n$ ) are clues for the detection of a transverse breathing crack in a rotating shaft, even if uncertainties are introduced in the rotor system. The same conclusions can be observed for the evolutions of the the mean value and the standard deviation of the non-linear horizontal response of the cracked rotor for the three cases (see Figures 8).

Moreover, it appears that increasing uncertainties of the rotor's stiffness and of the excitation forces affects the standard deviation of all the $n \times$ amplitudes when the rotor is passing through the $\frac{1}{n}$ subcritical speed. The range of speed where the non-linear amplitudes of the rotor system are predominant increase drastically with increasing uncertainties. This last observation clearly demonstrates that basic methods based on linear condition monitoring techniques (such as the changes in frequencies and modes shapes) can be rendered ineffective in the case of specific configurations taking into account uncertainties for the detection of cracks in rotor systems. However, whatever the levels and different kinds of uncertainties (such as material and/or loading characteristics) presented here, these numerical results demonstrate that emergences and variations in $n \times$ harmonic components of the system in the frequency domain are key indicators for the detection of transverse cracks in a rotating shaft. It is reminded for the reader comprehension that even if there is uncertainties in the value of stiffness property and unbalance, the evolution of damage will take a predictable path and the expected value of the $n \times$ amplitudes will be within a band of possible values due to uncertainties.

Finally Figures 9, 10,11 and 12 illustrate the mean values and the standard deviation of the nonlinear $n \times$ amplitudes (with $n=1,2,3$ and 4) for a rotor system containing a small crack (i.e. $\mu=0.5$ ). The previous results (with $\mu=1$ ) are also given for comparison. In this last part of the study, the random stiffness properties of shaft (i.e. for the Young modulus) and the excitation forces have each a variation coefficient equal to $2 \%$. As previously seen for $\mu=1$, the emergence of the $n \times$ amplitudes are clearly detected or $\mu=0.5$. So it may be concluded that using condition monitoring techniques based on non-linear approaches can be considered to be the first step for a rapid estimation of the presence of damage in rotors with structural uncertainties even if the crack size is small. Nevertheless, it is observed that the evolutions of the mean value and the standard deviation of 
the $1 \times$ harmonic components $(2 \times$ and $3 \times$ harmonic components, respectively $)$ are not significant at $\frac{1}{2}$ of the first critical speed ( $\frac{1}{3}$ and $\frac{1}{4}$ respectively). Showing Figures 9, it is observed a large difference in the mean values of the $1 \times$ amplitudes for two different crack depths in horizontal rotor response while almost no difference in the vertical rotor responses. Then, considering Figures 11 or 12, variations of the mean values of the $3 \times$ and $4 \times$ amplitudes are significant for both the vertical and horizontal directions. These results illustrate interaction between the contributions of the unbalance and the breathing of the crack.

Finally, it is shown that increasing the crack size induces a small decrease of the frequency values of the critical and sub-critical speeds that may be observed even if uncertainties of the rotor's stiffness and of the excitation forces are introduced. However, these changes in frequencies are quite small and can not be used for a robust detection of the presence of damage in rotors.

\section{Conclusion}

This paper presents an extension of the use of Harmonic Balance Method in the stochastic domain using the Polynomial Chaos Expansion to calculate the random non-linear dynamical response of mechanical systems. Here, the proposed methodology is applied to investigate the effects of the presence of a transverse crack in a rotating shaft under uncertain physical parameters in order to outline some robust indicators for detecting damage in rotating system. We considered the stiffness properties of shaft of the rotor system and the excitation forces random by Gaussian law. The random non-linear response consists in the mean and the standard deviation of the non-linear response and is obtained by finding the deterministic coefficients associated to the Polynomial Chaos Expansion applied on the $n \times$ harmonics which are given from the Harmonic Balance Method. The comparison of the results from this method and the reference one, combining the simulations of Monte Carlo and the Harmonic Balance Method, for one case in particular, validates the proposed methodology. Then a complete study is done for different cases of uncertainties (from $0 \%$ to $5 \%$ ) appearing on the stiffness properties of shaft of the rotor system and on the excitation forces to carry out the effect on the non-linear response. Besides, two values of depths were studied.

For a practical point of view, using condition monitoring techniques based on linear approaches (i.e. frequency shifts and changes in modal parameters) can be considered to be unpractical and not efficient for an estimation of the presence of damage if uncertainties are present in the mechanical systems. So alternative indicators based on the non-linear signature of dynamical systems can be more effective for a robust detection of the presence of damage in complex mechanical engineering structures. Even if uncertainties are introduced in real engineering structures (more particularly in rotating applications), an important observation is the recognition of the appearances of the $n \times$ harmonic components when the running speed reaches $\frac{1}{n}$ of the critical speeds. However due to interaction between the unbalance and the breathing of the crack, the variations of the mean and standard deviation of amplitudes for two different crack depths can be more significant in the horizontal direction than in the vertical direction. Moreover, in some cases, no difference for two different crack depths should be observed in the mean and standard deviation of $1 \times$ amplitudes if the unbalance is predominant versus the breathing of the crack. 


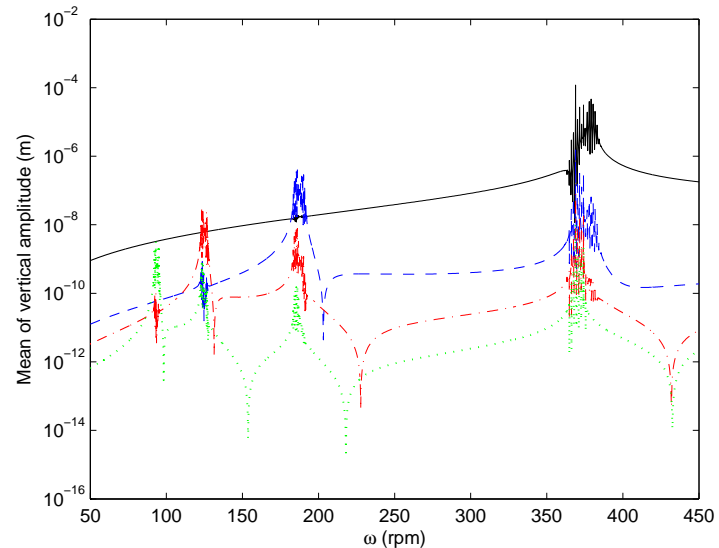

(a)

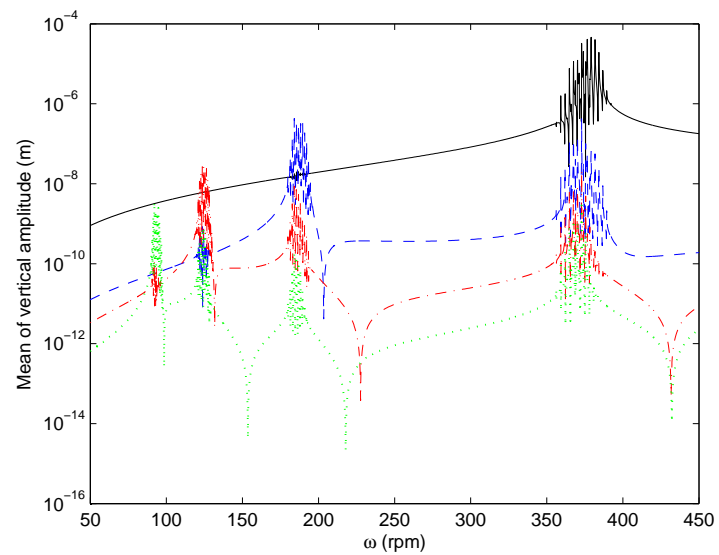

(c)

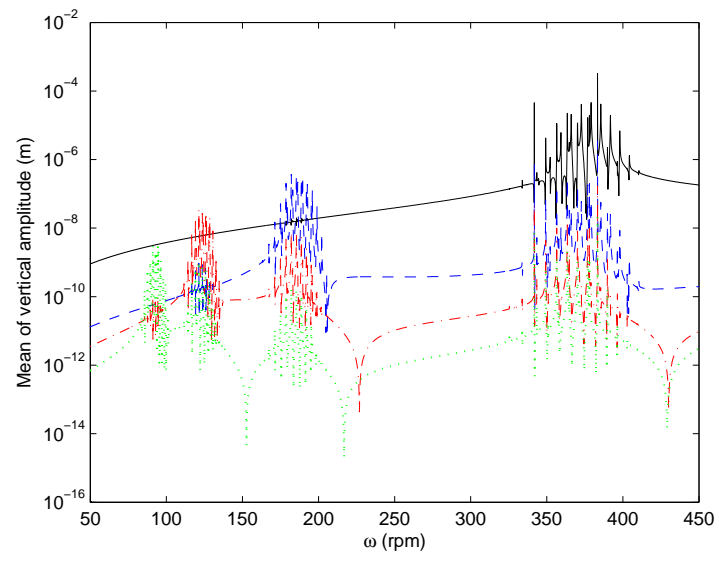

(e)

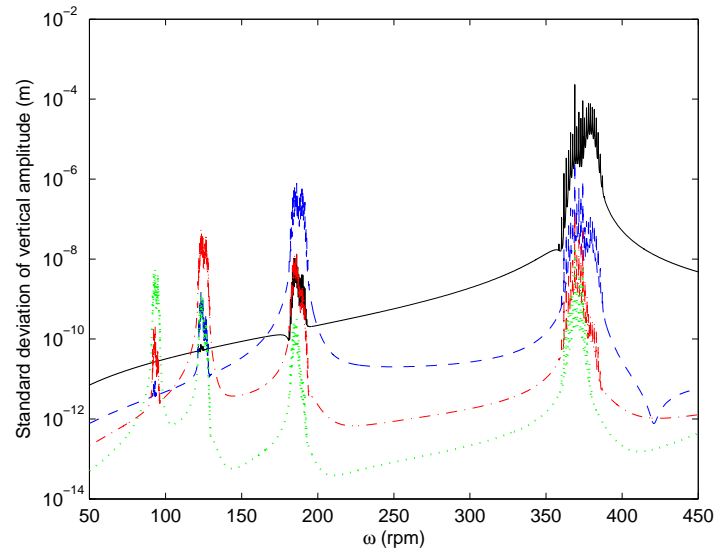

(b)

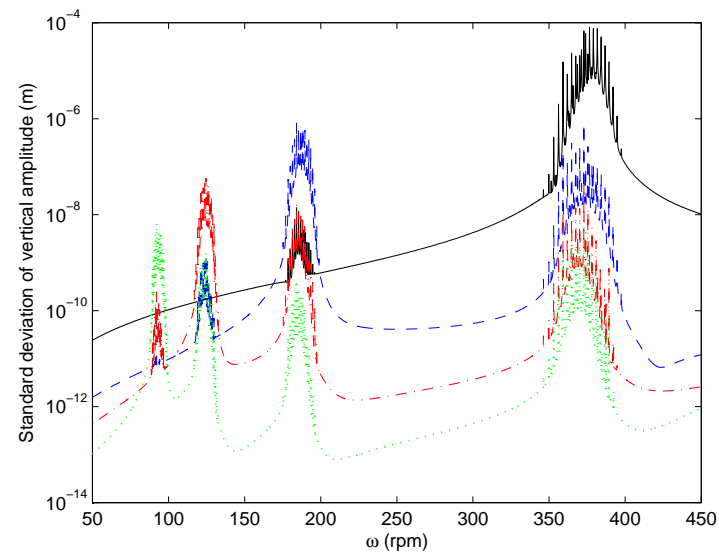

(d)

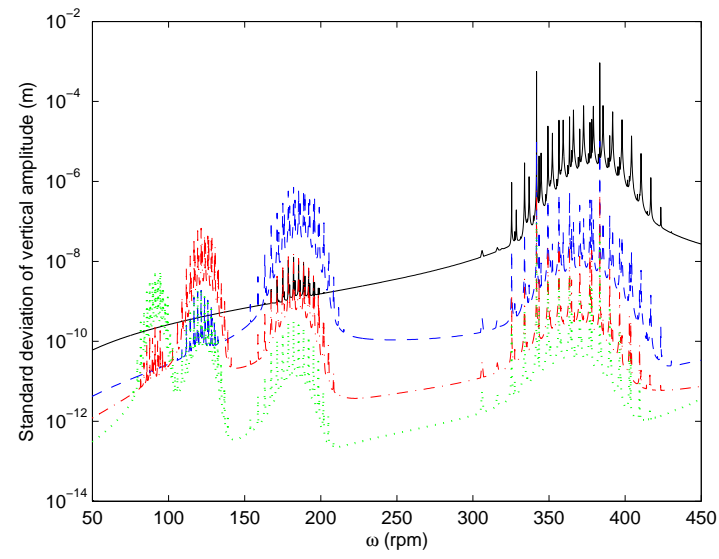

(f)

Figure 7: Evolution of the vertical $n \times$ displacements at the middle of the shaft for a non-dimensional crack depth $\mu=1$ with variations of the Young modulus and the excitation forces ( black=order 1 , - blue $=$ order $2,-$.red $=$ order $3, \cdots$ green $=$ order 4 ) (a) mean $-1 \%$ variations (b) standard deviation - $1 \%$ variations (c) mean - $2 \%$ variations (d) standard deviation - $2 \%$ variations (e) mean - $5 \%$ variations (f) standard deviation - 5\% variations 


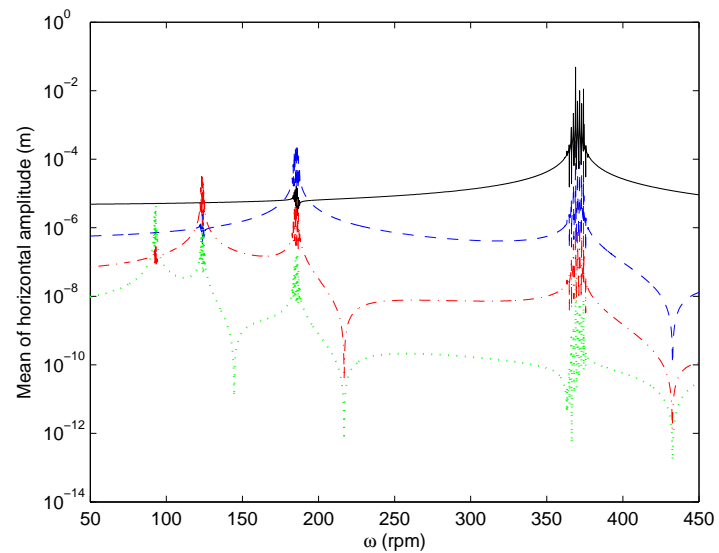

(a)

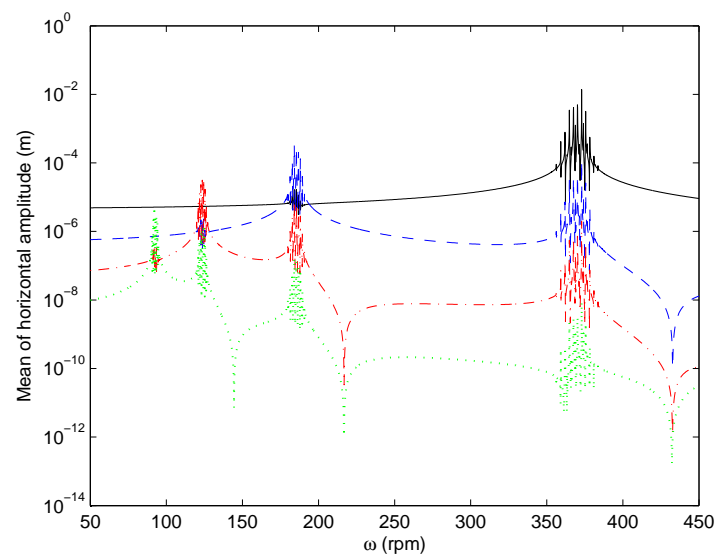

(c)

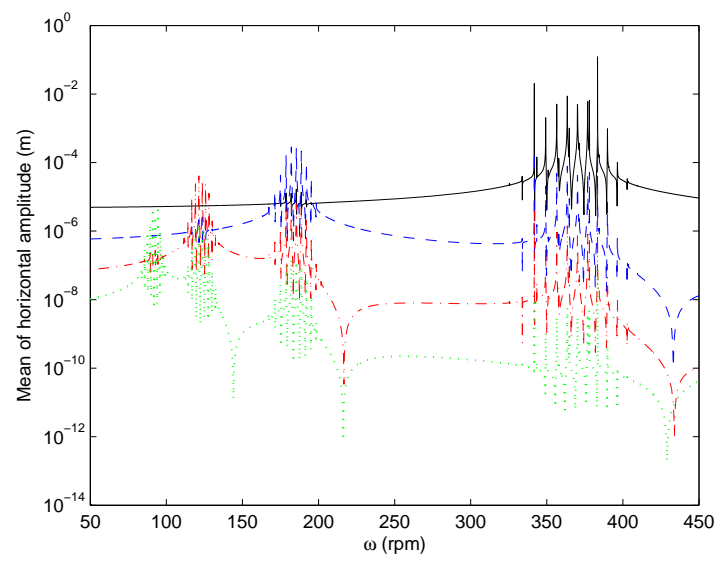

(e)

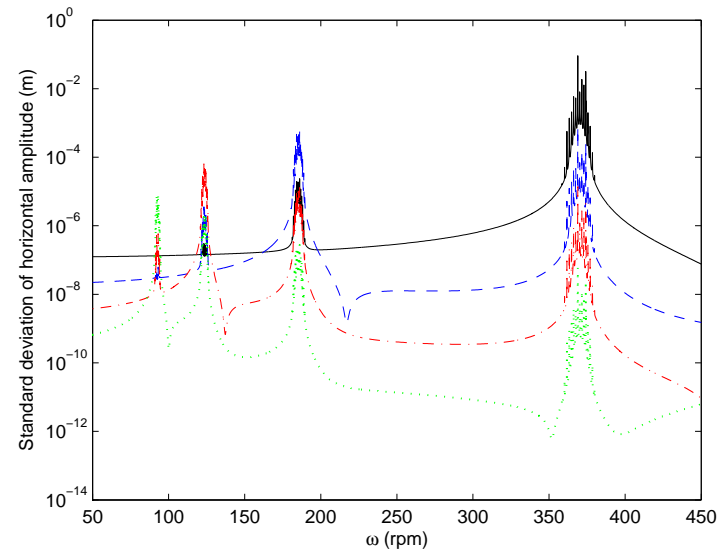

(b)

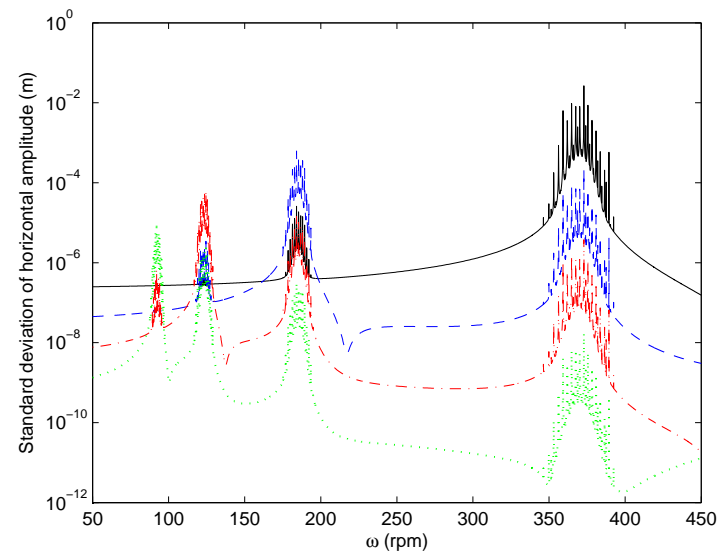

(d)

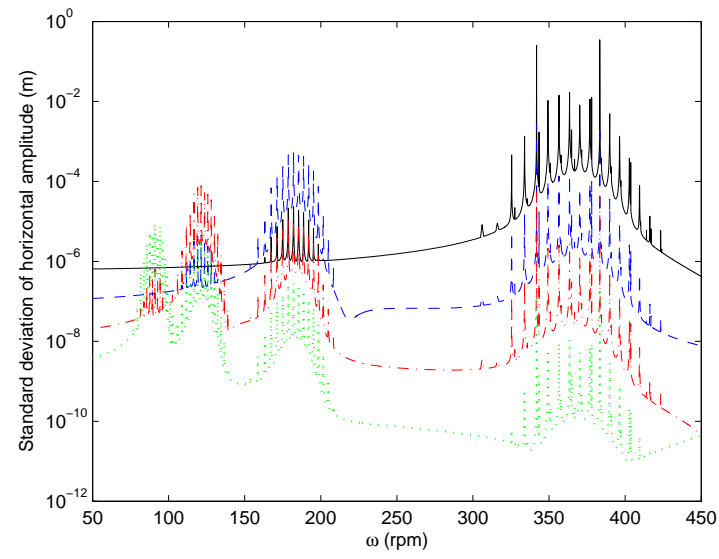

(f)

Figure 8: Evolution of the horizontal $n \times$ displacements at the middle of the shaft for a non-dimensional crack depth $\mu=1$ with variations of the Young modulus and the excitation forces ( black=order 1 , -- blue $=$ order $2,-$.red $=$ order $3, \cdots$ green $=$ order 4 ) (a) mean $-1 \%$ variations (b) standard deviation - $1 \%$ variations (c) mean - $2 \%$ variations (d) standard deviation - $2 \%$ variations (e) mean - $5 \%$ variations (f) standard deviation - 5\% variations 


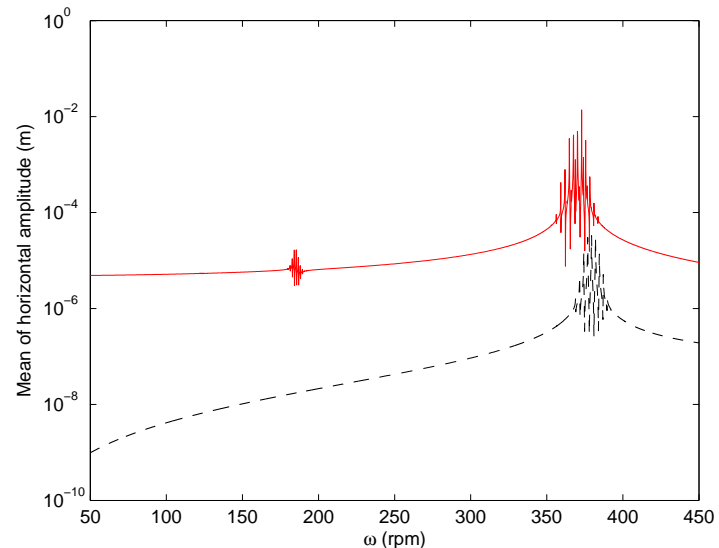

(a)

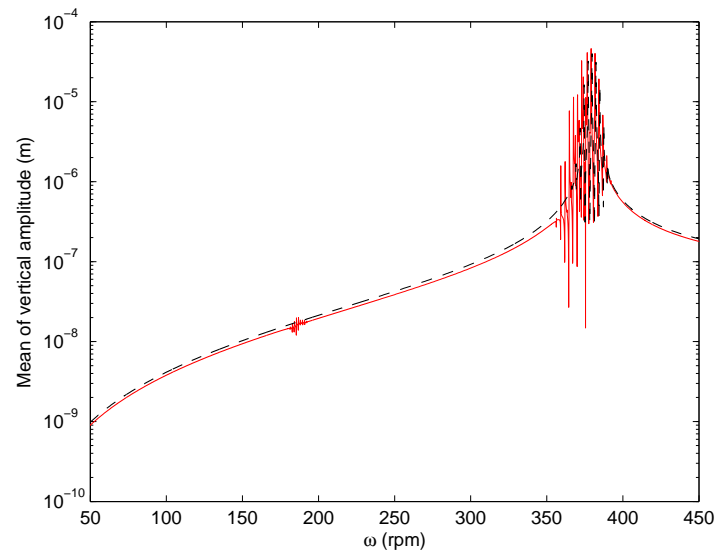

(c)

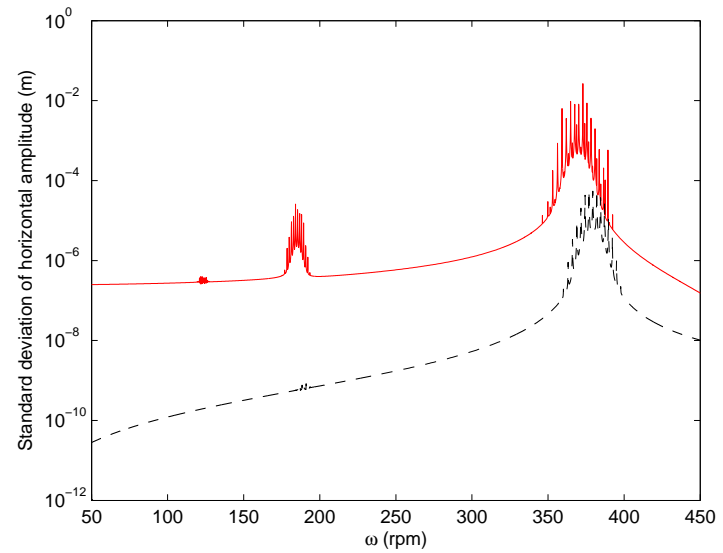

(b)

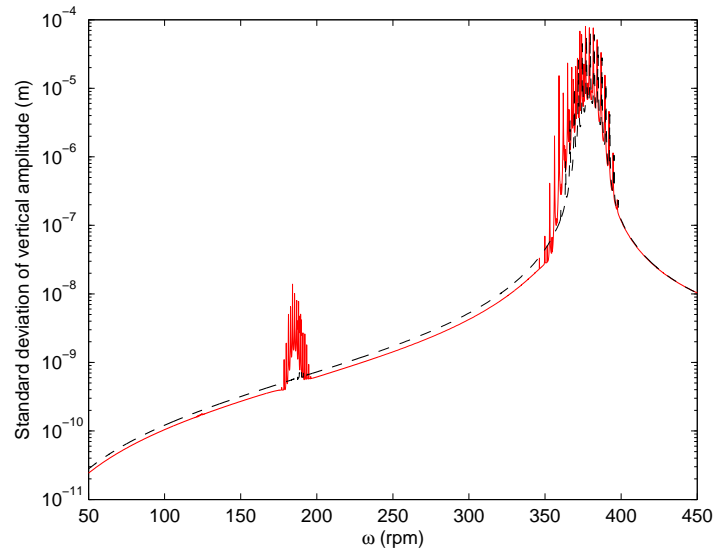

(d)

Figure 9: Evolution of the vertical and horizontal $1 \times$ displacements at the middle of the shaft for two crack sizes $(--$ black: $\mu=1$, red: $\mu=0.5)$ with $2 \%$ variations of the Young modulus and the excitation forces (a) mean of order 1 - horizontal (b) standard deviation of order 1 - horizontal (c) mean of order 1 - vertical (d) standard deviation of order 1 - vertical 


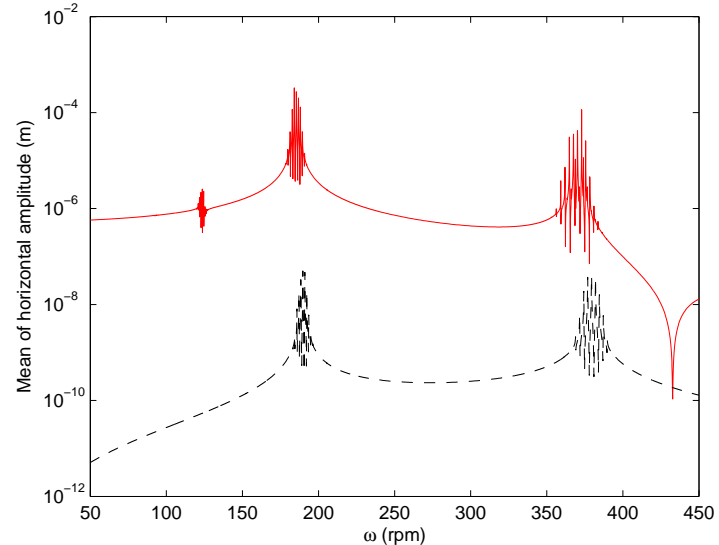

(a)

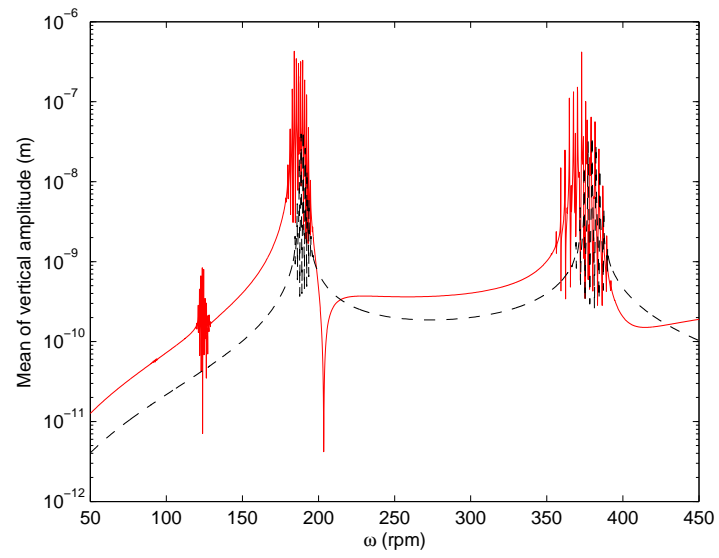

(c)

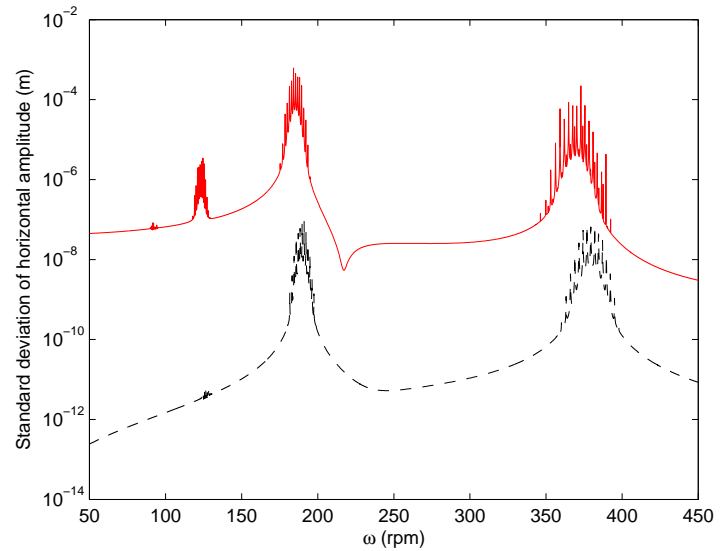

(b)

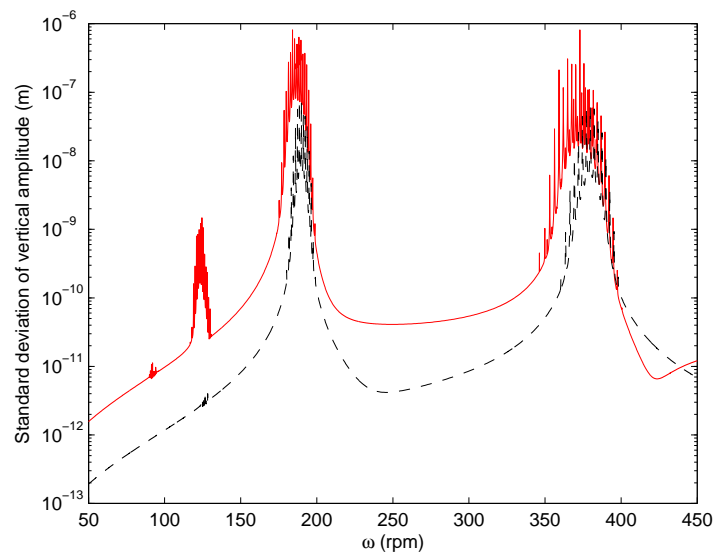

(d)

Figure 10: Evolution of the vertical and horizontal $2 \times$ displacements at the middle of the shaft for two crack sizes $(--$ black: $\mu=1$, red: $\mu=0.5)$ with $2 \%$ variations of the Young modulus and the excitation forces (a) mean of order 2 - horizontal (b) standard deviation of order 2 - horizontal (c) mean of order 2 - vertical (d) standard deviation of order 2 - vertical 


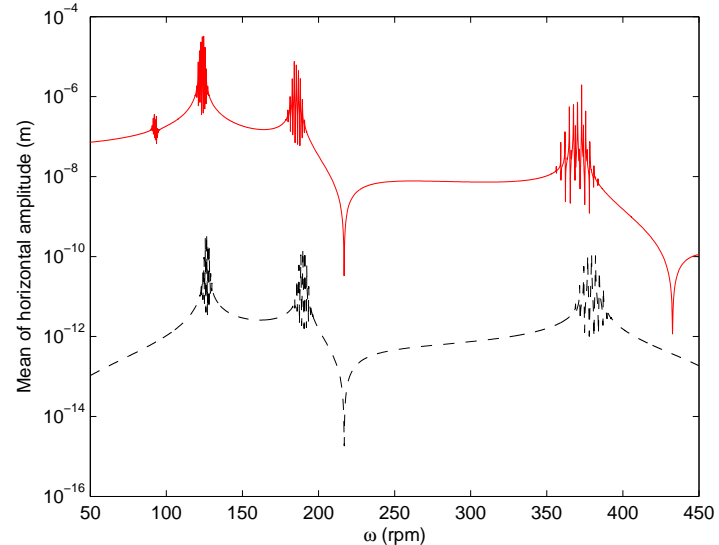

(a)

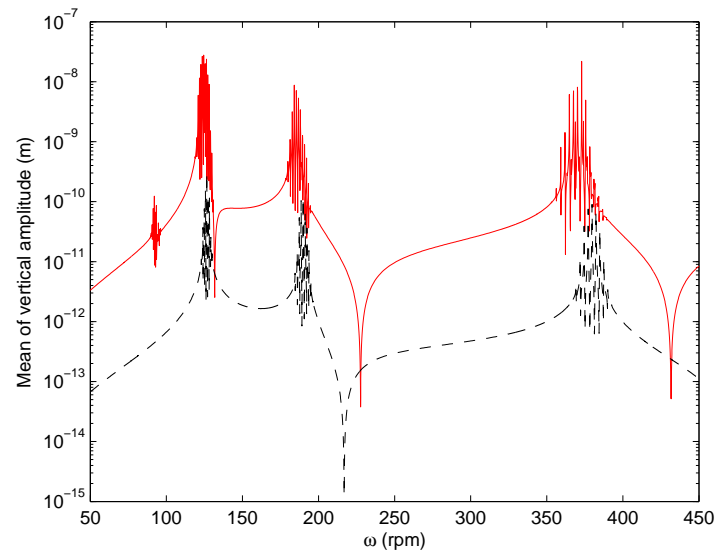

(c)

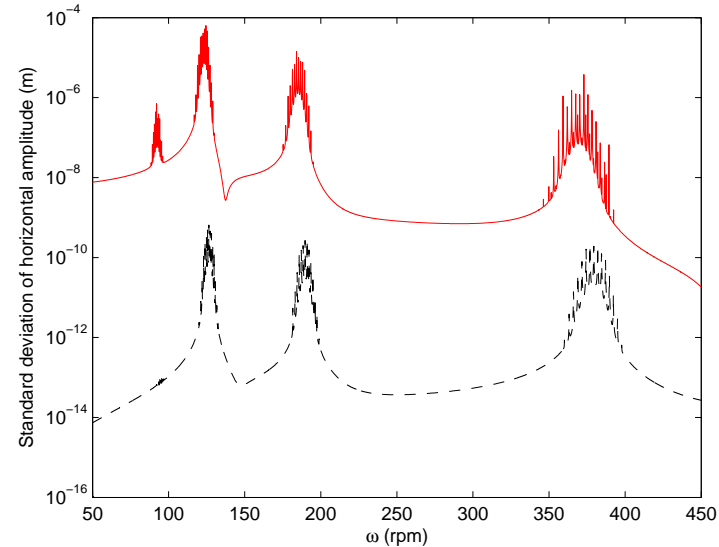

(b)

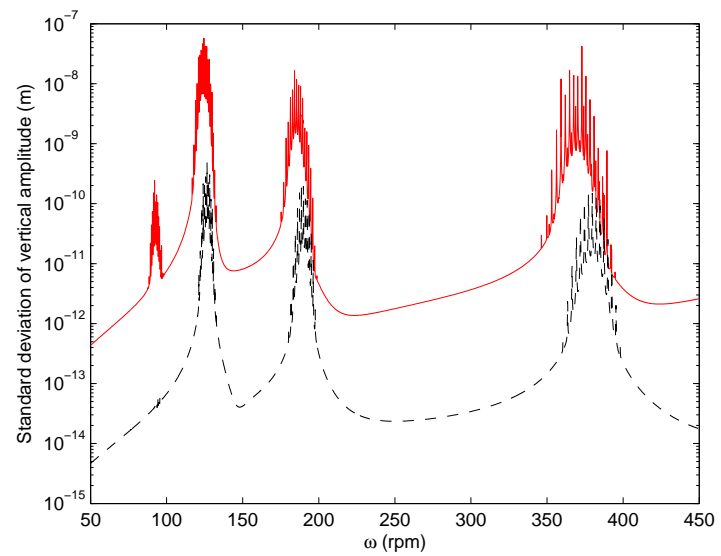

(d)

Figure 11: Evolution of the vertical and horizontal $3 \times$ displacements at the middle of the shaft for two crack sizes $(--$ black: $\mu=1$, red: $\mu=0.5)$ with $2 \%$ variations of the Young modulus and the excitation forces (a) mean of order 3 - horizontal (b) standard deviation of order 3 - horizontal (c) mean of order 3 - vertical (d) standard deviation of order 3 - vertical 


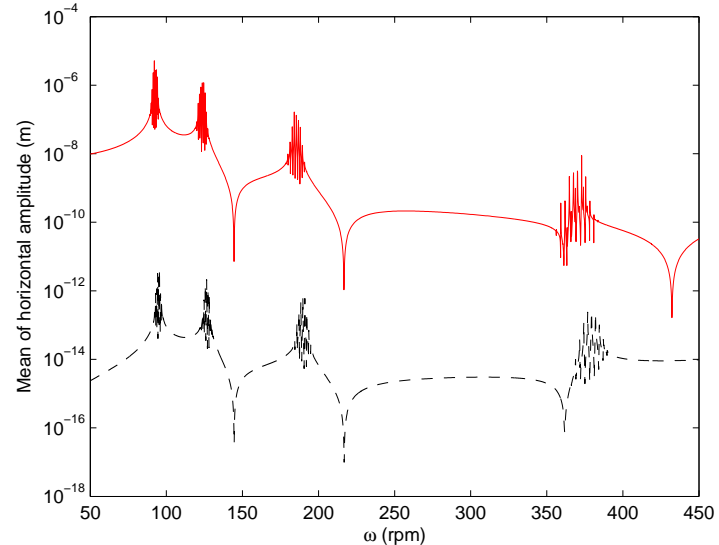

(a)

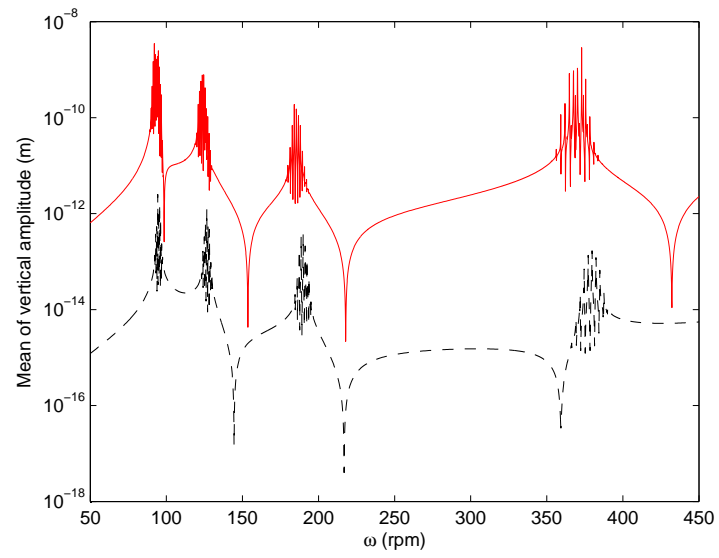

(c)

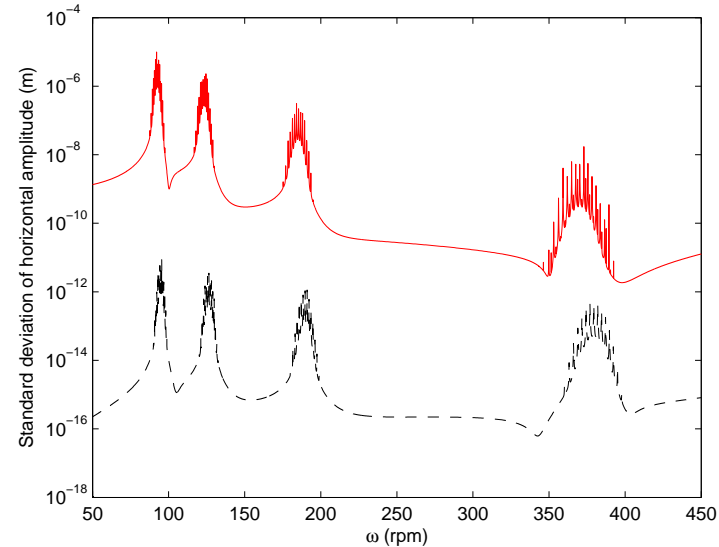

(b)

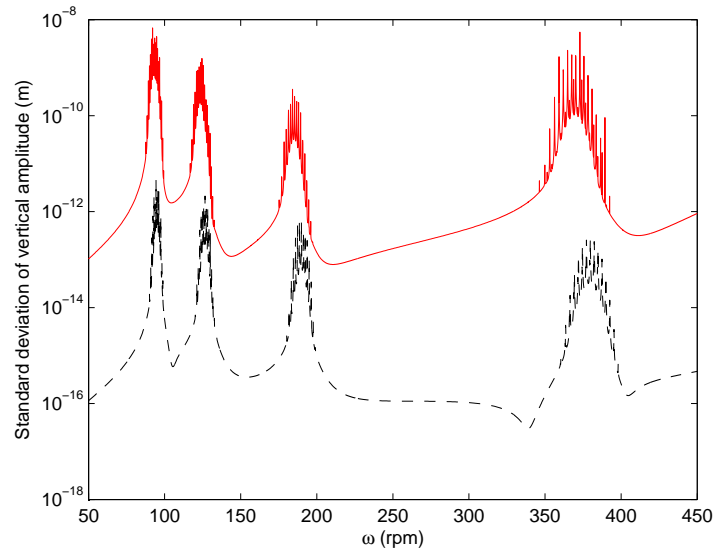

(d)

Figure 12: Evolution of the vertical and horizontal $4 \times$ displacements at the middle of the shaft for two crack sizes $(--$ black: $\mu=1$, red: $\mu=0.5)$ with $2 \%$ variations of the Young modulus and the excitation forces (a) mean of order 4 - horizontal (b) standard deviation of order 4 - horizontal (c) mean of order 4 - vertical (d) standard deviation of order 4 - vertical 


\section{References}

[1] Gasch, R., 1976. "Dynamic behaviour of a simple rotor with a cross-sectional crack". IMechE Conference on Vibrations in Rotating Machinery, C/178/76, pp. 123-128.

[2] Wauer, J., 1990. "Dynamics of cracked rotors: Literature survey". Applied Mechanics Review, 43, pp. $13-17$.

[3] Dimarogonas, A., 1996. "Vibration of cracked structures: a state of the art review". Engineering Fracture Mechanics, 55, pp. 831-857.

[4] Davies, W. G. R., and Mayes, I. W., 1984. "The vibrational behaviour of a multi-shaft, multibearing system in the presence of a propagating transverse crack". Transactions of the ASME Journal of Vibration, Acoustics, Stress, and Reliability in Design, 106, pp. 146-153.

[5] Mayes, I. W., and Davies, W. G. R., 1984. "Analysis of the response of a multi-rotor-bearing system containing a transverse crack in a rotor". Transactions of the ASME Journal of Vibration, Acoustics, Stress, and Reliability in Design, 106, pp. 139-145.

[6] Salawu, O., 1997. "Detection of structural damage through changes in frequency: a review". Engineering Structures, 19(9), pp. 718-723.

[7] Gasch, R., 1993. "A survey of the dynamic behaviour of a simple rotating shaft with a transverse crack". Journal of Sound and Vibration, 160(2), pp. 313-332.

[8] Sinou, J.-J., 2008. "Detection of cracks in rotor based on the $2 \mathrm{x}$ and 3x super-harmonic frequency components and the crack-unbalance interactions". Communications in Nonlinear Science and Numerical Simulation, 13(9), pp. 2024-2040.

[9] Adewusi, S., and Al-Bedoor, B., 2001. "Wavelet analysis of vibration signals of an overhang rotor with a propagating transverse crack". Journal of Sound and Vibration, 246(5), pp. 777-793.

[10] Adewusi, S., and Al-Bedoor, B., 2002. "Experimental study on the vibration of an overhung rotor with a propagating transverse crack". Shock and Vibration, 9, p. 91-104.

[11] Sekhar, A. S., 2003. "Crack detection and monitoring in a rotor supported on fluid film bearings: start-up vs run-down". Mechanical Systems and Signal Processing, 17(4), p. 897-901.

[12] Sekhar, A., 2003. "Crack detection through wavelet transform for a run-up rotor". Journal of Sound and Vibration, 259, p. 461-472 .

[13] Zou, J., and Chen, J., 2004. "A comparative study on time-frequency feature of cracked rotor by wigner-ville distribution and wavelet transform". Journal of Sound and Vibration, 276, p. 1-11.

[14] Sinou, J.-J., 2009. "An experimental investigation of condition monitoring for notched rotors through transient signals and wavelet transform". Experimental Mechanics, 49, p. 683-695.

[15] Gómez-Mancilla, J., Sinou, J.-J., Nosov, V., Thouverez, F., and Zambrano, A., 2004. "The influence of crack-imbalance orientation and orbital evolution for an extended cracked jeffcott rotor". Comptes Rendus Mecanique, 332(12), pp. 955-962. 
[16] Bachschmid, N., Pennacchi, P., and Tanzi, E., 2008. "Some remarks on breathing mechanism, on non-linear effects and on slant and helicoidal cracks". Mechanical Systems and Signal Processing, 22, p. 879-904.

[17] Sinou, J.-J., and Lees, A. W., 2005. "Influence of cracks in rotating shafts". Journal of Sound and Vibration, 285(4-5), pp. 1015-1037.

[18] Sinou, J.-J., and Lees, A. W., 2007. "A non-linear study of a cracked rotor". European Journal of Mechanics A/Solids, 26(1), pp. 152-170.

[19] Machorro-López, J.-M., Adams, D.-E., Gomez-Mancilla, J.-C., and Gul, K.-A., 2009. "Identification of damaged shafts using active sensing - simulation and experimentation". Journal of Sound and Vibration, 327(3-5), pp. 368-390.

[20] Sinou, J.-J., 2009. "Experimental study on the nonlinear vibrations and nx amplitudes of a rotor with a transverse crack". Journal of Vibration and Acoustics, 131,041008, pp. 1-6.

[21] Darpe, A., Gupta, K., and Chawla, A., 2004. "Transient response and breathing behaviour of a cracked jeffcott rotor". Journal of Sound and Vibration, 272, p. 207-243.

[22] Darpe, A., Gupta, K., and Chawla, A., 2002. "Analysis of the response of a cracked jeffcott rotor to axial excitation". Journal of Sound and Vibration, 249, p. 429-445.

[23] Darpe, A., Gupta, K., and Chawla, A., 2004. "Coupled bending, longitudinal and torsional vibrations of a cracked rotor". Journal of Sound and Vibration, 269, p. 33-60.

[24] Darpe, A., 2007. "Coupled vibrations of a rotor with slant crack". Journal of Sound and Vibration, 305, p. 172-193.

[25] Loève, M., 1977. Perturbation Methods, $4^{\text {th }}$ edition. Springer-Verlag.

[26] Benaroya, H., and Rehak, M., 1988. "Finite element methods in probabilistic structural analysis: A selective review". Applied Mechanics Reviews, 41(5), pp. 201-213.

[27] Yamazaki, F., Shinozuka, M., and Dasgupta, G., 1988. "Neumann expansion for stochastic finite element analysis". Journal of Engineering Mechanics ASCE, 114(8), pp. 1335-1354.

[28] Ghanem, R., and Spanos, P., 1991. Stochastic Finite Elements: A Spectral Approach. SpringerVerlag.

[29] Wiener, N., 1938. "The homogeneous chaos". Am J Math, 60, pp. 897-936.

[30] Ghanem, R., 1999. "Stochastic finite elements with multiple random non- gaussian properties". J Eng Mech, 125, pp. 26-40.

[31] Soize, C., and Ghanem, R., 2004. "Physical systems with random uncertainties: Chaos representations with arbitrary probability measure". SIAM J. Sci. Comput., 26 (2), pp. 395-410.

[32] Sinou, J.-J., 2007. "Effects of a crack on the stability of a non-linear rotor system". International Journal of Non-Linear Mechanics, 42, p. 959 - 972.

[33] Nayfeh, A., 1973. Probability Theory, $4^{\text {th }}$ edition. John Wiley and Sons, London. 
[34] Dessombz, O., 2000. Analyse dynamique de structures comportant des paramètres incertains. PhD Thesis, Ecole Centrale de Lyon, France. 